\title{
Molecular gas kinematics in the nuclear region of nearby Seyfert galaxies with ALMA
}

\author{
A. Bewketu Belete ${ }^{1}$, P. Andreani ${ }^{2}$, J. A. Fernández-Ontiveros ${ }^{3}$, E. Hatziminaoglou ${ }^{2}$, F. Combes ${ }^{4}$, M. Sirressi ${ }^{5}$,
} R. Slater ${ }^{6,7}$, C. Ricci ${ }^{8}$, K. Dasyra ${ }^{9,10}$, C. Cicone $^{11}$, S. Aalto ${ }^{12}$, L. Spinoglio ${ }^{3}$, M. Imanishi ${ }^{13}$, and J. R. De Medeiros ${ }^{1}$

${ }^{1}$ Departamento de Física Teórica e Experimental, Universidade Federal do Rio Grande do Norte, Natal, RN 59078-970, Brazil e-mail: asnakew@fisica.ufrn.br

2 European Southern Observatory, Karl-Schwarzschild-Straße 2, 85748 Garching, Germany e-mail: pandrean@eso.org

3 Istituto di Astrofisica e Planetologia Spaziali (INAF-IAPS), Via Fosso del Cavaliere 100, 00133 Roma, Italy

4 Observatoire de Paris, LERMA, College de France, PSL University, CNRS, Sorbonne University, 75014 Paris, France

5 Department of Astronomy and Oskar Klein Centre, Stockholm University, AlbaNova 106 91, Stockholm, Sweden

${ }^{6}$ Departamento de Astronomía, Universidad de La Serena, Av. Juan Cisternas 1200, La Serena, Chile

7 Dirección de Formación General, Facultad de Educación y Cs. Sociales, Universidad Andres Bello, Sede Concepción, Talcahuano, Chile

8 Núcleo de Astronomía, Facultad de Ingeniería y Ciencias Universidad Diego Portales (UDP), Santiago de Chile, Chile

9 Dept. of Astrophysics, Astronomy \& Mechanics, Faculty of Physics, National and Kapodistrian University of Athens, Panepis-timiopolis, Zografou 15784, Greece

10 National Observatory of Athens, Institute for Astronomy, Astrophysics, Space Applications and Remote Sensing, Penteli 15236, Athens, Greece

11 Institute of Theoretical Astrophysics, University of Oslo, PO Box 1029, Blindern 0315, Oslo, Norway

12 Chalmers University of Technology, Department of Earth and Space Sciences, Onsala Space Observatory, 43992 Onsala, Sweden

13 National Astronomical Observatory of Japan 2-21-1 Osawa, Mitaka, Tokyo 181-8588, Japan

Received 3 February 2021 / Accepted 12 May 2021

\section{ABSTRACT}

Context. The study of the distribution, morphology, and kinematics of cold molecular gas in the nuclear and circumnuclear regions of active galactic nuclei (AGNs) helps to characterise and hence to quantify the impact of the AGNs on the host galaxy over its lifetime. Aims. We present the analysis of the molecular gas in the nuclear regions of three Seyfert galaxies, NGC 4968, NGC 4845, and MCG06-30-15, using Atacama Large sub-Millimetre Array (ALMA) observations of the $\mathrm{CO}(2-1)$ emission line. The aim is to determine the kinematics of the gas in the central $(\sim 1 \mathrm{kpc})$ region and thereby to probe nuclear fueling and feedback of AGNs.

Methods. We used two different softwares, namely the 3D-Based Analysis of Rotating Object via Line Observations and DiskFit, to model the kinematics of the gas in the molecular disc, and thereby to determine the gas rotation and any kinematical perturbations. Results. Circular motions dominate the kinematics of the molecular gas in the central discs, mainly in NGC 4845 and MCG-06-30-15; however there is clear evidence of non-circular motions in the central ( 1 kpc) region of NGC 4845 and NGC 4968. The strongest non-circular motion is detected in the inner disc of NGC 4968, mainly along the minor kinematic axis, with a velocity $\sim 115 \mathrm{~km} \mathrm{~s}^{-1}$. Of all DiskFit models, the bisymmetric model is found to give the best fit for NGC 4968 and NGC 4845, indicating that the observed non-circular motions in the inner disc of these galaxies could result from the nuclear barred structure, where the gas streams in elliptical orbits aligned along the bar. If the dynamics of NGC 4968 is modelled as a corotation pattern just outside of the bar, the bar pattern speed becomes $\Omega_{\mathrm{b}}=52 \mathrm{~km} \mathrm{~s}^{-1} \mathrm{kpc}^{-1}$; the corotation is set at $3.5 \mathrm{kpc}$; and the inner Lindblad resonance (ILR) ring is $R=300 \mathrm{pc}$, corresponding to the CO emission ring. In the NGC 4968 galaxy, the torques exerted on the gas by the bar are positive in the centre, within the gas nuclear ring, and negative outside. This shows that the gas is transiently trapped in the ILR. The comparison of the CO intensity maps with the map of the cold dust emission shows an absence of CO in the centre of NGC 4968; also the dust distribution and $\mathrm{CO}$ emission in and around the centre of NGC 4845 have similar extensions. The $1.2 \mathrm{~mm}$ ALMA continuum is peaked and compact in NGC 4968 and MCG-06-30-15, but their $\mathrm{CO}(2-1)$ emissions have extended distributions. Allowing the $\mathrm{CO}-$ to- $\mathrm{H}_{2}$ conversion factor $\alpha_{\mathrm{CO}}$ between 0.8 and 3.2, which is typical of nearby galaxies of the same type, the molecular mass $M\left(\mathrm{H}_{2}\right)$ is estimated to be $\sim 3-12 \times 10^{7} M_{\odot}(\mathrm{NGC} 4968), \sim 9-36 \times 10^{7} M_{\odot}$ (NGC 4845), and $\sim 1-4 \times 10^{7} M_{\odot}$ (MCG-06-30-15).

Conclusions. We conclude that the observed non-circular motions in the molecular disc of NGC 4968 and likely those seen in NGC 4845 are due to the presence of the bar in the nuclear region. We discuss the possibility that the observed pattern in the kinematics might be a consequence of the presence of AGNs, and this might be the case for NGC 4845. At the current spectral and spatial resolution and sensitivity, we cannot claim any strong evidence in these sources of the long sought feedback or feeding effect resulting from the presence of AGNs.

Key words. galaxies: structure - ISM: kinematics and dynamics - galaxies: individual: NGC 4968 galaxies: individual: NGC 4845 - galaxies: individual: MCG-06-30-15 


\section{Introduction}

Powerful active galactic nuclei (hereafter, AGNs) can drive fast outflows that affect the properties and kinematics of the ambient gas in the nuclear and circumnuclear regions of their host galaxies. By heating the gas and expelling it from the central regions, AGNs may affect the star formation histories of the host galaxy bulge (see e.g. Fluetsch et al. 2019; Oosterloo et al. 2017; Fiore et al. 2017; Morganti 2017 and references therein). Even though the mechanisms that can produce molecular outflows are still uncertain (Morganti 2017), cold molecular gas is known as the phase that dominates the central regions and is found to be the most massive outflow component in AGNs (see e.g. Kanekar \& Chengalur 2008; Cicone et al. 2014; Dasyra \& Combes 2011, 2012; Morganti et al. 2015; Feruglio et al. 2010; Rupke \& Veilleux 2013; García-Burillo et al. 2015; Carniani et al. 2015; Fiore et al. 2017; Morganti 2017; Fluetsch et al. 2019), thus making cold molecular gas the main tracer of AGN outflows.

Several works aim to understand the origin of non-circular motions, outflow driving mechanisms, and the impact of AGN feedback on the interstellar medium (ISM) by imaging and modelling the kinematics of cold molecular gas in the central regions of host galaxies of AGNs. Both types of non-circular motions (inflows and outflows) are detected in the nuclear and circumnuclear regions of nearby star-forming and AGN host galaxies. Using the ALMA observations of dense molecular gas tracers $(\mathrm{CO}(3-2), \mathrm{CO}(6-5), \mathrm{HCN}(4-3), \mathrm{HCO}+(4-3)$, and $\mathrm{CS}(7-6))$ García-Burillo et al. (2014) studied the fueling and feedback of star formation and nuclear activity in the nearby Seyfert 2 nucleus NGC 1068.

The authors confirm the detection of molecular line and dust continuum emissions from different regions in the source. They also indicate the presence of an inward radial flow in the starburst ring and bar region as well as a massive outflow that is an order of magnitude higher than the star formation rate (SFR) in the inner region $(r \sim 50 \mathrm{pc}$ out to $r \sim 400 \mathrm{pc})$. The authors interpret the inward flow as the combined action of the bar and spiral arms, and the outflow is AGN driven. Based on the tight correlation between the ionised gas outflow, radio jet, and occurrence of outward motions in the disc, the authors suggest that the outflow is likely AGN driven.

With a better spatial resolution of $\sim 4 \mathrm{pc}$ towards the same galaxy, García-Burillo et al. (2016) studied the dust emission and the distribution and kinematics of molecular gas by using the ALMA observations of the dust continuum at $432 \mu \mathrm{m}$ and the $\mathrm{CO}(6-5)$ molecular line emission in its circumnuclear disc. These authors conclude that the overall slow rotation pattern of the disc is perturbed by strong non-circular motions and enhanced turbulence (see also a similar work in García-Burillo et al. 2019).

Combes et al. (2014) report the ALMA observations of $\mathrm{CO}(3-2)$ emission in the nuclear region of the Seyfert 1 galaxy NGC 1566. They find a conspicuous nuclear trailing spiral and weak non-circular motions at the periphery of the nuclear spiral arms. Recently, by analysing the nuclear kinematics of the same galaxy NGC 1566 via ALMA observations of the $\mathrm{CO}(2-1)$ emission, Slater et al. (2019) show the presence of significant non-circular motions in the innermost $(200 \mathrm{pc})$ and along spiral arms in the central kpc. They find a molecular outflow in the disc with velocities of $\sim 180 \mathrm{~km} \mathrm{~s}^{-1}$ in the nucleus.

Using the ALMA observations of $\mathrm{CO}(3-2)$ emission around the nucleus of NGC 1433, Combes et al. (2013) also find an intense high-velocity $\mathrm{CO}$ emission feature red-shifted to $200 \mathrm{~km} \mathrm{~s}^{-1}$, with a blue-shifted counterpart, at $2^{\prime \prime}(100 \mathrm{pc})$ from the centre, interpreting the wide component as an outflow partly driven by the central star formation, but mainly boosted by the AGN through its radio jets. Similarly, using the ALMA observations of the infrared-luminous merger NGC 3256, Sakamoto et al. (2014) detect high-velocity molecular outflows from the northern and southern nucleus with two different large velocities $>750 \mathrm{~km} \mathrm{~s}^{-1}$ and $[1000 \sim 2000] \mathrm{km} \mathrm{s}^{-1}$, respectively, interpreting the northern outflow as a starburstdriven superwind and the southern one as an outflow driven by a bipolar radio jet from an AGN.

In their ALMA $\mathrm{CO}(2-1)$ line observations with angular resolutions $0.11^{\prime \prime}-0.26^{\prime \prime}$ (9-21 pc), Alonso-Herrero et al. (2018) find strong non-circular motions in the central $\left(0.2^{\prime \prime}-0.3^{\prime \prime}\right)$ regions of the nearby Seyfert galaxy NGC 5643 with velocities of up to $110 \mathrm{~km} \mathrm{~s}^{-1}$, explaining the motions as radial outflows in the nuclear disc in the absence of a nuclear bar. Domínguez-Fernández et al. (2020) present a detailed analysis of the kinematics and morphology of cold molecular gas in the nuclear/circumnuclear regions of five nearby $(19-58 \mathrm{Mpc})$ Seyfert galaxies, Mrk 1066, NGC 2273, NGC 4253, NGC 4388, and NGC 7465. The authors detect $\mathrm{CO}(2-1)$ emission in all galaxies with disky or circumnuclear ring like morphologies. Moreover, they find in all galaxies, though the bulk of the gas is rotating in the plane of the galaxy, non-circular motions in four of the galaxies (Mrk 1066, NGC 4253, NGC 4388 and NGC 7465). They interpret the non-circular motions in NGC 4253, NGC 4388, and NGC 7465 as streaming motions due to the presence of a large-scale bar, whereas the non-circular motions in the nuclear regions of Mrk 1066 and NGC 4388 are outflows due to the interaction of the AGN wind with molecular gas in the galaxy disc.

Fast and massive molecular outflows are capable of impacting the nearby ISM, and molecular mass outflow rate is shown to be correlated well with AGN bolometric luminosity (Fiore et al. 2017). Fiore et al. (2017) also report that in AGNs with bolometric luminosity up to $\sim 10^{46} \mathrm{erg} \mathrm{s}^{-1}$ the molecular mass outflow rate dominates its ionised counterpart. Moreover, Fiore et al. (2017) find that the molecular gas depletion timescale and the molecular gas fraction of galaxies hosting powerful AGN driven winds are 3-10 times shorter and smaller than those of main sequence galaxies with similar SFRs, stellar masses, and redshifts, indicating that at high AGN bolometric luminosity the reduced molecular gas fraction may be the result of the destruction of molecules by the wind, leading to a larger fraction of gas in the atomic ionised phase.

Also using the ALMA observations of $\mathrm{CO}(2-1)$, Morganti et al. (2015) find that the gas kinematics in the Seyfert 2 galaxy IC 5063 are very complex. The authors detect fast, cold molecular gas outflow with velocities up to $650 \mathrm{~km} \mathrm{~s}^{-1}$, indicating that the outflow can be driven by the central AGN and the radio jet. Similarly, using the $\mathrm{CO}(2-1)$ and $\mathrm{CO}(3-2)$ ALMA observations of the nearby Seyfert 1.5 galaxy NGC 3227, Alonso-Herrero et al. (2019) show the presence of CO clumps with complex kinematics, dominated by non-circular motions in the central region $\left(1^{\prime \prime} \sim 73 \mathrm{pc}\right)$. Fernández-Ontiveros et al. (2020) also show the presence of a prominent jet-driven outflow of $\mathrm{CO}(2-1)$ molecular gas along the kinematic minor axis of the Seyfert 2 galaxy ESO 420-G13 at a distance of 340-600 pc from the nucleus.

Several similar works were published on constraining the kinematics and dynamics of molecular gas in the nuclear or circumnuclear regions of star-forming and AGN host galaxies (e.g. Tadhunter et al. 2014; Ramakrishnan et al. 2019; Salak et al. 2020; Lutz et al. 2020; Combes et al. 2019; Audibert et al. 2019; Sirressi et al. 2019, see also review by Veilleux et al. 2020). 
In this paper, we present the analysis of the $\mathrm{CO}$ molecular gas kinematics in the nuclear regions of three different types of Seyfert galaxies, NGC 4968, NGC 4845, and MCG-06-30-15, using the ALMA observation of the bright $\mathrm{CO}(2-1)$ emission line as a tracer. These galaxies are different in AGN type, AGN luminosity, and morphology. Knowledge about the nature of cold molecular gas kinematics and its origin in the nuclear and circumnuclear regions of such different AGN host galaxies is essential to verify whether the driving mechanism(s) is the same; this in turn could be used as an input to construct a universal model that describes such physical mechanism(s).

The three galaxies belong to the Twelve micron WInd STatistics (TWIST) sample (Fernández-Ontiveros et al. 2020, and in prep.), which aims to study 41 AGNs extracted from the IRAS $12 \mu \mathrm{m}$ flux-limited sample (Rush et al. 1993). These sources are located close enough $(10<D<50 \mathrm{Mpc})$ to ensure a good spatial resolution through ALMA observations and to resolve the morphological structures in the central $\sim 3 \times 3 \mathrm{kpc}$ region. The sources are above the so-called knee of the Seyfert galaxy luminosity function; therefore, they are statistically representative to determine the impact of the outflow and inflow mechanisms for the bulk of the AGN population. Moreover the sample has been extensively studied in the past 30 years, and there is a wealth of ancillary data, from X-ray to radio wavelengths, on this sample (Fernández-Ontiveros et al., in prep.). The three galaxies in this work extend the preliminary study of the TWIST sample initiated in Fernández-Ontiveros et al. (in prep.) for ESO 420G13. These include one type 1 and two type 2 nuclei located at 20-40 Mpc, which sample three different bins in X-ray luminosity. Table 1 lists the properties of the three objects under study.

To study the molecular gas kinematics we use two different softwares, namely the 3D-Based Analysis of Rotating Object via Line Observations (3D BAROLO ${ }^{1}$; Di Teodoro \& Fraternali 2015) and DiskFit (Peters \& Kuzio de Naray 2017). This paper is structured as follows. In Sect. 2 we present a brief description of the properties of the sources, the ALMA observations, and data reduction. We discuss modelling of the $\mathrm{CO}$ molecular gas kinematics in Sect. 3. The properties and kinematics of the CO molecular gas, and the residuals in each galaxy are presented in Sects. 4-6. We discuss the results in Sect. 7, and the main conclusions in Sect. 8.

We adopt a flat $\Lambda$ CDM cosmology with $\Omega_{\lambda}=0.7, \Omega_{\lambda}=0.3$, and $H_{0}=70 \mathrm{~km} \mathrm{~s}^{-1} \mathrm{Mpc}^{-1}$.

\section{Basic galaxy properties and observations}

NGC 4968 is a nearby Seyfert 2 spiral galaxy classified as a barred spiral (SB0) according to the Hubble and de Vaucouleurs galaxy morphological classification. This galaxy is located at a redshift of $z=0.00986$ (LaMassa et al. 2017; Malkan et al. 1998), corresponding to a distance of $42 \mathrm{Mpc}$. The NGC 4968 narrow line region (NLR) was imaged by Ferruit et al. (2000) in the [O III] $\lambda 5007,[\mathrm{~N} \mathrm{II}] \lambda \lambda 6548,6583$, and $\mathrm{H} \alpha$ emission lines, as well as in its adjacent continua (centred near 5500 and $8000 \AA$ ) using the Hubble Space Telescope (HST). From the continuum images these authors estimate a photometric major axis PA of $45^{\circ}$ and an inclination angle (INC) of $60^{\circ}$ at radii greater than $10^{\prime \prime}(2.1 \mathrm{kpc})$ (Schmitt et al. 2003, see also). The NLR extends towards the south-east side of the nucleus and the [OIII] line emission has a wedge-shaped morphology filling the

\footnotetext{
1 https://bbarolo.readthedocs.io/en/latest/
}

Table 1. Physical and geometrical parameters of the galaxies.

\begin{tabular}{llcc}
\hline \hline & NGC 4968 & NGC 4845 & MCG-6-30-15 \\
\hline AGN type & S2 & S2 & S1.2 \\
Redshift & 0.00986 & 0.00411 & 0.008 \\
Classification & SB0 & SABab & Sab \\
$\log L_{2-10 \mathrm{keV}}$ & 43.20 & 41.98 & 42.74 \\
$\left(\mathrm{erg} \mathrm{s}^{-1}\right)$ & & & \\
$\log L_{12 \mu \mathrm{m}}$ & 9.91 & 9.87 & 9.52 \\
$\left(L_{\odot}\right)$ & & & \\
SFR $\left(M_{\odot} \mathrm{yr}^{-1}\right)$ & 4.29 & - & 1.82 \\
Distance & $42 \mathrm{Mpc}$ & $18 \mathrm{Mpc}$ & $37 \mathrm{Mpc}$ \\
RA & $13 \mathrm{~h} 07 \mathrm{~m} 05.935 \mathrm{~s}$ & $12 \mathrm{~h} 58 \mathrm{~m} 01.187 \mathrm{~s}$ & $13 \mathrm{~h} 35 \mathrm{~m} 53.777 \mathrm{~s}$ \\
Dec & $-23^{\circ} 40^{\prime} 36.23^{\prime \prime}$ & $1^{\circ} 34^{\prime} 32.526^{\prime \prime}$ & $-34^{\circ} 17^{\prime} 44.242^{\prime \prime}$ \\
INC $\left(^{\circ}\right)$ & 60 & 73 & {$[65-68]$} \\
PA $\left(^{\circ}\right)$ & {$[234-256]$} & {$[62-81]$} & {$[115-122]$} \\
\hline
\end{tabular}

Notes. The SFR is computed from PAH (polycyclic aromatic hydrocarbon) observations (Mordini et al., in prep.) and $12 \mu \mathrm{m}$ luminosity in $\left[\log _{10} L_{\odot}\right]$ (from Rush et al. 1993). The X-ray luminosity is from Brightman \& Nanda (2011).

cavity inside the dusty ring. The presence of the dust may be responsible for its morphology, but the presence of a ionisation cone projecting against the far side of the galaxy disc cannot be excluded (Ferruit et al. 2000).

Strong et al. (2004) studied the properties of the molecular gas in NGC 4968 with single dish (15-m) millimetre observations at the ESO-SEST telescope. These authors estimate the line luminosity $\left(L_{\mathrm{CO}}\right)$ and molecular mass $\left(M\left(\mathrm{H}_{2}\right)\right)$ through observations from $\mathrm{CO}(1-0)\left(6.0 \times 10^{7} \mathrm{~K} \mathrm{~km} \mathrm{~s}^{-1} \mathrm{pc}^{2}\right.$ and $21.0 \times 10^{7} M_{\odot}$, respectively), and $\mathrm{CO}(2-1)\left(4 \times 10^{7} \mathrm{~K} \mathrm{~km} \mathrm{~s}^{-1} \mathrm{pc}^{2}\right.$ and $15.0 \times$ $10^{7} M_{\odot}$, respectively), making use of a CO-to- $\mathrm{H}_{2}$ conversion factor, $\alpha_{\mathrm{CO}}$, of 3.47 .

The galaxy NGC 4845 is a nearby Seyfert 2 with a clear starburst component (Thomas et al. 2017), classified as an intermediate barred spiral galaxy (SABab), and is located in the Virgo Southern Extension at $z=0.00411$ (Irwin et al. 2015 and references therein). We adopt the Tully-Fisher distance of $18 \mathrm{Mpc}$. The inclination on the sky is almost edge-on, revealing contrasted dust lanes on the near side and a peanut-shape for the bulge. The galaxy contains a bright unresolved core with a surrounding weak central disc $(1.8 \mathrm{kpc}$ diameter). The radio spectrum of the core has been known to evolve with time, which could be due to an adiabatic expansion (outflow), likely in the form of a jet or cone (Irwin et al. 2015). Using spectral observations of NGC 4845 in different position angles $\left(\mathrm{PA}=44^{\circ}, 78^{\circ}\right.$, $98^{\circ}, 118^{\circ}$, and $178^{\circ}$ ), Bertola et al. (1989) studied the kinematics of the ionised gas in the central region $(r \leq 1.5 \mathrm{kpc})$ and revealed a regular but non-axisymmetric velocity field. Based on photometry, Bertola et al. (1989) also point out the presence of a possible slight twisting between the disc and bulge isophotes, interpreting this as an indication of a triaxial bulge in NGC 4845. This galaxy shows an ionisation cone with an opening angle of $120^{\circ}$ perpendicular to the circumstellar disc of HII regions; the counter-ionisation cone is faint, likely because of extinction (Thomas et al. 2017).

The early-type Sab galaxy MCG-06-30-15 is a Seyfert 1.2 galaxy (Makarov et al. 2014) located at a distance of $37 \mathrm{Mpc}, z=0.008$ (Wu et al. 2009). This active galaxy has a $400 \mathrm{pc}$ diameter stellar kinematically distinct core (KDC) that is counter-rotating with respect to the main body of the galaxy (Raimundo et al. 2017). The molecular gas, traced by the $\mathrm{H}_{2}$ $2.12 \mu \mathrm{m}$ emission is also counter-rotating with respect to the 
main stellar body of the galaxy, implying that the formation of the distinct core is associated with the inflow of external gas into the centre of MCG-6-30-15 and that the event that formed the counter-rotating core is also the main mechanism providing gas for the fuelling of the AGN. Moreover, this shows that external gas accretion is able to significantly replenish the fuelling reservoir in such active galaxies. MCG-06-30-15 NLR was imaged using HST by Ferruit et al. (2000) in the [O III] $\lambda 5007$ and [N II] $\lambda \lambda 6548,6583$ and $\mathrm{H} \alpha$ emission lines, as well as in their adjacent continua (centred near 5500 and $8000 \AA$ ). Ferruit et al. (2000) estimated the photometric major axis PA and an INC of $115^{\circ}$ and $60^{\circ}$, respectively. The [O III] image reveals a nuclear extension aligned parallel to the photometric major axis of the galaxy, which presumably represents gas that is coplanar with the stellar disc (see also Schmitt et al. 2003).

Rosario et al. (2018) measure the $\mathrm{CO}(2-1)$ single dish flux and discuss the properties of its molecular gas in relation to its AGN properties. These authors estimate the line luminosity $\left(L_{\mathrm{CO}}\right)$ and molecular mass $\left(M\left(\mathrm{H}_{2}\right)\right)$ through observations from $\mathrm{CO}(2-1)\left(1.3 \times 10^{7} \mathrm{~K} \mathrm{~km} \mathrm{~s}^{-1} \mathrm{pc}^{2}\right.$ and $1.4 \times 10^{7} M_{\odot}$, respectively $)$ using a CO-to- $\mathrm{H}_{2}$ conversion factor, $\alpha_{\mathrm{CO}}$, of 1.1.

\subsection{ALMA observations}

The observations of the bright $\mathrm{CO}(2-1)$ line at $230.5 \mathrm{GHz}$ rest frequency (in Band 6) were carried out as part of ALMA cycle 5 under project IDs 2017.1.00236.S in December 2017 and January 2018. With a spatial resolution between $25 \mathrm{pc}$ and $48 \mathrm{pc}$, the $\mathrm{CO}(2-1)$ maps are sensitive enough to detect molecular masses as low as $\sim 10^{5} M_{\odot}(5 \sigma)$ per beam. See the summary of ALMA observations in Table 1.

\subsection{Data reduction and imaging}

Data were calibrated and post-processed using the Common Astronomy Software Applications (CASA) package (McMullin et al. 2007), applying the standard calibration recipes provided by the ALMA observatory. The data were calibrated using the CASA version 4.7.0 and the calibration script provided by the observatory, while further post-processing was done using the CASA version 5.4.0. In all cases, the hogbom deconvolution algorithm was applied using briggs weighting and a robustness value 2.0 to reconstruct the final data cubes, thereby optimising the value of the limiting flux threshold in each case. The spectral data cubes for the emission lines were produced with a channel width of $\sim 10$ and $\sim 30 \mathrm{~km} \mathrm{~s}^{-1}$ and a pixel size of about one-fifth to one-fourth of the synthesised beam size (see Table 1 for the synthesised beam size). The emission line regions were automatically masked during the cleaning process in the spectral cubes using the auto-multithresh algorithm in tclean.

The continuum emission was then subtracted in the spatial frequency domain, that is prior to the image reconstruction, using a 1D polynomial interpolation between the adjacent continuum channels at both sides of the respective emission lines. Continuum maps were constructed using all the continuum channels in the four spectral windows, that is discarding the channels with $\mathrm{CO}(2-1)$ emission. The masking procedure for the continuum data was run interactively during the cleaning process.

Finally, all data cubes were corrected for the primary beam attenuation pattern. Using the final $\mathrm{CO}(2-1)$ data cubes, the first three moments corresponding to the integrated intensity map of the line, the average velocity field and the average velocity dispersion map were computed numerically. In order to reduce the
Table 2. ALMA CO(2-1) observation log.

\begin{tabular}{llll}
\hline \hline \multicolumn{4}{l}{ Target line CO(2-1) } \\
\hline & NGC 4968 & NGC 4845 & MCG-06-30-15 \\
\hline$v_{\text {rest }}$ & $230.5 \mathrm{GHz}$ & $230.5 \mathrm{GHz}$ & $230.5 \mathrm{GHz}$ \\
Date & Dec 2017 & Dec 2017 & Dec 2017 \\
& Jan 2018 & Jan 2018 & Jan 2018 \\
Array configuration & C43-5 & C43-5 & C43-5 \\
Spatial resolution & $48 \mathrm{pc}$ & $45 \mathrm{pc}$ & $25 \mathrm{pc}$ \\
Channel width & $2.5 \mathrm{~km} \mathrm{~s}^{-1}$ & $2.5 \mathrm{~km} \mathrm{~s}^{-1}$ & $2.5 \mathrm{~km} \mathrm{~s}^{-1}$ \\
rms sensitivity & $1.4 \mathrm{mJy} \mathrm{beam}^{-1}$ & $7 \mathrm{mJy} \mathrm{beam}^{-1}$ & $0.7 \mathrm{mJy} \mathrm{beam}^{-1}$ \\
Synthesised beam & $0.27^{\prime \prime} \times 0.22^{\prime \prime}$ & $0.56^{\prime \prime} \times 0.48^{\prime \prime}$ & $0.16^{\prime \prime} \times 0.13^{\prime \prime}$ \\
\hline
\end{tabular}

noise in the moment maps, for each spaxel only those channels with a signal-to-noise ratio $(\mathrm{S} / \mathrm{N})$ above $\sim 5$ the median absolute deviation were considered, following the approach in Fernández-Ontiveros et al. (2020).

\section{Modelling the kinematics of $\mathrm{CO}(2-1)$ emission line}

\subsection{D modelling of the main rotating disc}

We use the $\mathrm{CO}(2-1)$ emission line as a tracer of the main kinematic features of the $\mathrm{CO}$ gas in the nuclear region of the galaxies. To constrain the gas kinematical perturbations we construct a 3D disc model using the 3D BAROLO software and fit the model to the $\mathrm{CO}(2-1)$ emission line data cube. This software automatically fits 3D tilted-ring models to emission-line data cubes. The model assumes that the emitting material at each radius is confined within a geometrically thin disc and its kinematics are dominated by pure rotational motion (Di Teodoro \& Fraternali 2015). The model requires the geometrical parameters of the disc, namely the centre position coordinates, the INC, and the kinematic PA. When the emission peaks at the centre, such as in the case of NGC 4845, the coordinates can be determined by fitting a 2D Gaussian to the central part (where the peak emission is) of the intensity map (see Sirressi et al. 2019); whereas for maps without a central emission peak (the case of NGC 4968 and MCG-06-30-15) the code uses a source-finder algorithm to identify the emission region, and then calculates the geometrical centroid, weighted by the flux intensity. The centre positions are determined and reported in Table 2. The INC, the angle between the normal to the disc axis and the line of sight, can be inferred from the zeroth moment map or from the first moment map, depending on how well these moments can be estimated. For NGC 4845 we determine the INC parameter from the ratio of the two axes, major and minor axes, in the first moment (velocity) map (see e.g. Sirressi et al. 2019). But for well-resolved data (the case of NGC 4968) it is better to use the zeroth moment (intensity) map rather than the velocity field. The INC turns out to be $60^{\circ}$ (NGC 4968) by fitting an ellipse to the outer ring in the intensity map. For NGC 4845, the INC $=73^{\circ}$ is determined from the velocity field. For MCG-06-30-15, 3D BAROLO estimates that the INC angle varies from $65^{\circ}$ to $68^{\circ}$.

The 3D BAROLO programme measures the PA as the angle between the north and the receding half of the major axis (=positive velocities in the velocity field), measured anticlockwise (see Di Teodoro \& Fraternali 2015). The PA varies from $234^{\circ}$ to $256^{\circ}$ (NGC 4968) and from $62^{\circ}$ to $81^{\circ}$ (NGC 4845). For MCG-06-30-15, 3D BAROLO estimates that the PA varies from $115^{\circ}$ to $122^{\circ}$. 
Using the estimated geometrical parameters and assuming a number of rings at different radial separation we construct a disc model via the 3D BAROLO kinematic model and fit its emission to the continuum-subtracted ALMA $\mathrm{CO}(2-1)$ line data cube. We use the ALMA $\mathrm{CO}(2-1)$ line data cube with a channel widths of $10 \mathrm{~km} \mathrm{~s}^{-1}$.

The number of rings can be determined from the spatial extension of the object. The model uses ten rings with a radial separation of 0.217 arcsec. All the rings are placed at $N * R A D S E P+R A D S E P / 2$, where $N$ is the number of rings and $R A D S E P$ is the radial separation in arcsec.

To avoid the noise effect at the edges, 3D BAROLO measures the noise at the spectral channel edges when it builds the mask to avoid the noise effect at the edges instead of using the continuum-subtracted ALMA $\mathrm{CO}(2-1)$ line data cube with a velocity range $=(-1000,990) \mathrm{km} \mathrm{s}^{-1}$. Therefore, we consider the central regions containing the emission of the galaxies, reducing the velocity range of the input NGC 4968 data cube to $(-340,340) \mathrm{km} \mathrm{s}^{-1}$. To construct the disc model for NGC 4968, we use 27 rings with a radial separation of 0.12 arcsec.

The velocity range of the input NGC 4845 data cube is reduced to $(-510,240) \mathrm{km} \mathrm{s}^{-1}$ and we use 22 rings with a radial separation of 0.55 arcsec. The Barolo fits for $\mathrm{N} 4845$ for the velocity and the velocity dispersion are not optimal; however, the results do not improve by letting the IA vary between 73 and $80^{\circ}$.

For MCG-06-30-15, we feed the continuum-subtracted line data cube to the model without reducing its velocity range. The model is able to find the best model by reducing the residuals at minimum. This could be because the kinematics is purely dominated by regular rotation pattern (i.e. a simple kinematics). A slight deviation appears between the blue and red contours in $p-v$ diagram. The Barolo fits for N4845 for the velocity and velocity dispersion are not optimal; however, the results do not improve by letting the INC to vary between 73 and $80^{\circ}$.

The results from 3D BAROLO, the kinematic maps, and $p-v$ diagrams, are given in Figs. 1-3 for NGC 4968, NGC 4845, and MCG-06-30-15, respectively. The intensity maps of NGC 4968 and MCG-06-30-15 reveal the molecular gas distribution in the nuclear regions as a ring-like morphology. The gas distribution in the NGC 4845 appears to have an edge-on inclination.

\subsection{DiskFit modelling}

The DiskFit software fits simple axisymmetric and nonaxisymmetric non-parametric models either to photometric images or to kinematic maps (velocity fields) of disc galaxies. DiskFit fits an entire velocity field with a physically motivated model. The underlying assumption is that the circular orbit of a region of gas is affected by higher order perturbations (i.e. 'harmonics'). Physically, $m=1$ harmonics correspond to 'lopsided' perturbations and $m=2$ harmonics correspond to bisymmetric (i.e. bar) perturbations. The model is given by Peters \& Kuzio de Naray (2017), i.e.

$V_{\mathrm{m}}=V_{\mathrm{s}}+\sin i\left[V_{\mathrm{t}} \cos (\theta)-V_{\mathrm{m}, \mathrm{t}} \cos \left(\theta_{\mathrm{b}}\right) \cos (\theta)-V_{\mathrm{m}, \mathrm{r}}\left(\sin 2 \theta_{\mathrm{b}}\right) \sin (\theta)\right]$,

where $V_{\mathrm{m}}$ is the model (with $m$ specifying the harmonic order with $m=1$ or $m=2$ in the disc plane), $V_{\mathrm{s}}$ is the systemic velocity, $V_{\mathrm{t}}$ is the mean orbital speed, $\theta$ is the angle between a point in the disc relative to the major axis, $\theta_{\mathrm{b}}$ is the angle between a point in the disc relative to the bar, $V_{\mathrm{m}, \mathrm{t}}$ and $V_{\mathrm{m}, \mathrm{r}}$ are the tangential and radial components of the non-circular motions, $\theta$ and $\theta_{\mathrm{b}}$ are the

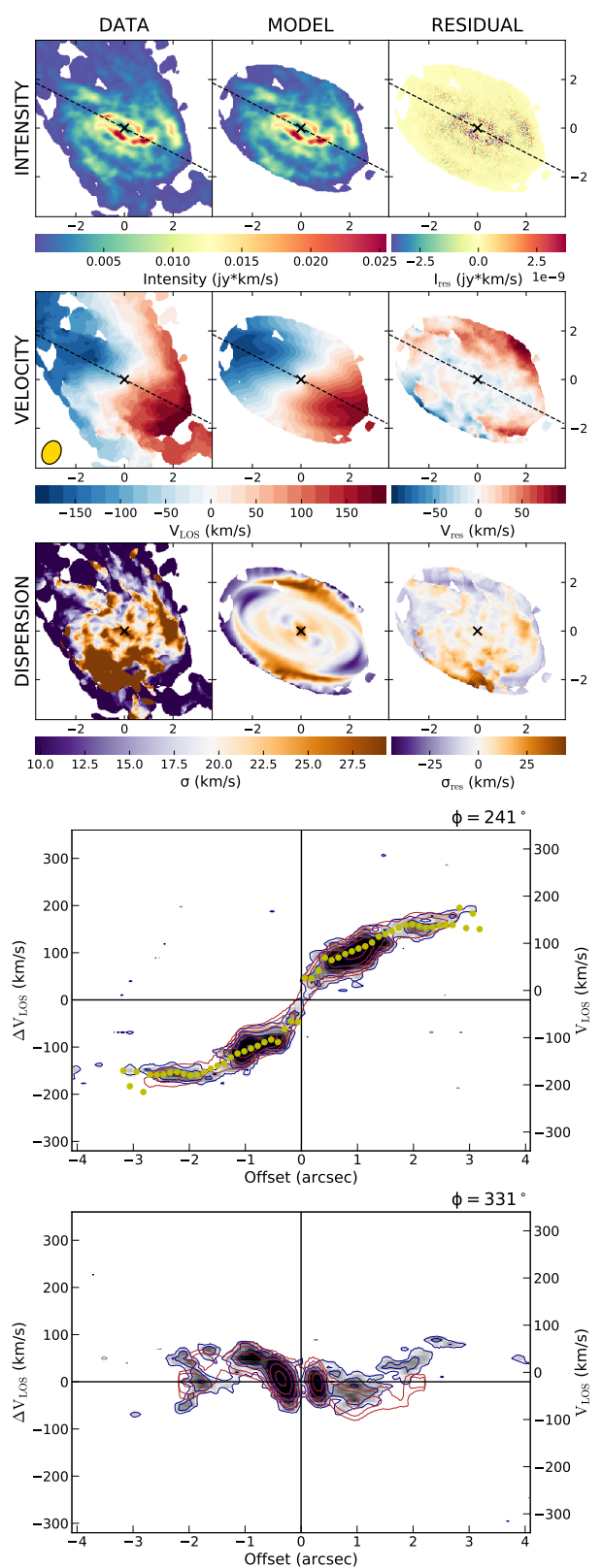

Fig. 1. Top panels: (from top to bottom; left panels): zeroth (intensity), first (velocity field), and second (velocity dispersion) moment maps of the ALMA CO(2-1) data of NGC 4968. Central panels: same as the left panels but for the best-fit model constructed with 3D BAROLO. Right panels: same as the left panels but for the residual (data model). The black cross denotes the centre of the galaxy. The major axis of the molecular disc is shown by a dashed black line in the velocity map. The yellow ellipse in the bottom left corner of the data velocity map shows the synthesised beam size $\left(0.27^{\prime \prime} \times 0.22^{\prime \prime}\right)$ with $\mathrm{PA}=245^{\circ}$. North and east directions correspond to top and left, respectively. Bottom panels: $p-v$ diagrams extracted from the data cube (solid blue contours) and model cube (solid red contours) along the major axis (top panel) and along the minor axis (bottom panel). The contours level of both the data and the model are at $[1,2,4,8,16,32,64]^{*} l$, where $l=0.0012$. The rotation velocity of each ring of the best-fit disc model is represented by the solid yellow dots in the top panel.

azimuthal angles relative to the major axis and the non-circular flow axis, respectively, and $i$ is the disc inclination. DiskFit can also fit radial flows (with $m=0$ distortions to the flow in the disc plane) and symmetric warps in the outer disc. 

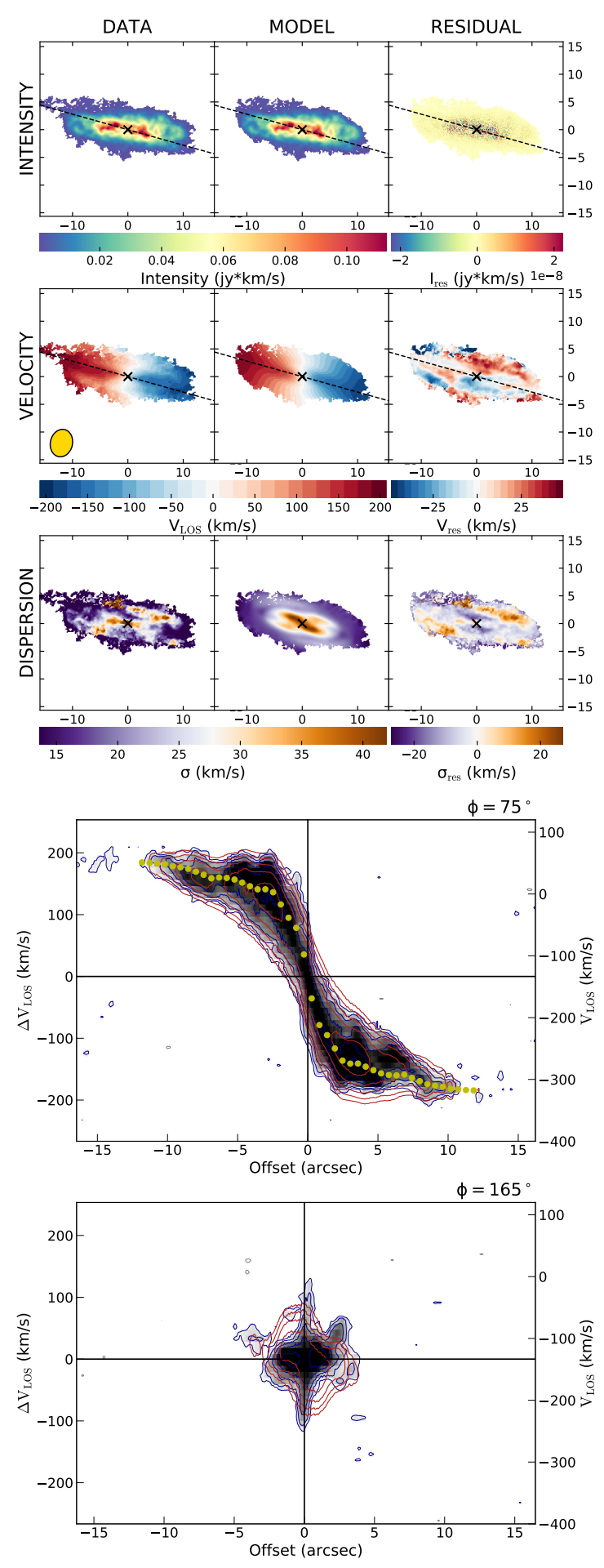

Fig. 2. Same as in Fig. 1 but for NGC 4845. The synthesised beam size $\left(0.56^{\prime \prime} \times 0.48^{\prime \prime}\right)$ is plotted in yellow in the bottom left corner of the data velocity map with $\mathrm{PA}=77^{\circ}$. The contours level of both the data and the model are at $[1,2,4,8,16,32,64]^{*} l$, where $l=0.00393635$.

DiskFit requires a flat inner disc, but it allows for a symmetric warp in the outer disc. The disc is assumed to be flat out to a warp radius $r_{\mathrm{w}}$, beyond which both the ellipticity and the PA of the line of nodes vary in proportion to $\left(r-r_{\mathrm{W}}\right)^{2}$. In addition, DiskFit can find the best-fitting inner warp radius $r_{\mathrm{w}}$ and peak change in ellipticity and PA of the line of nodes, or it can hold any combination of these parameters fixed. For an
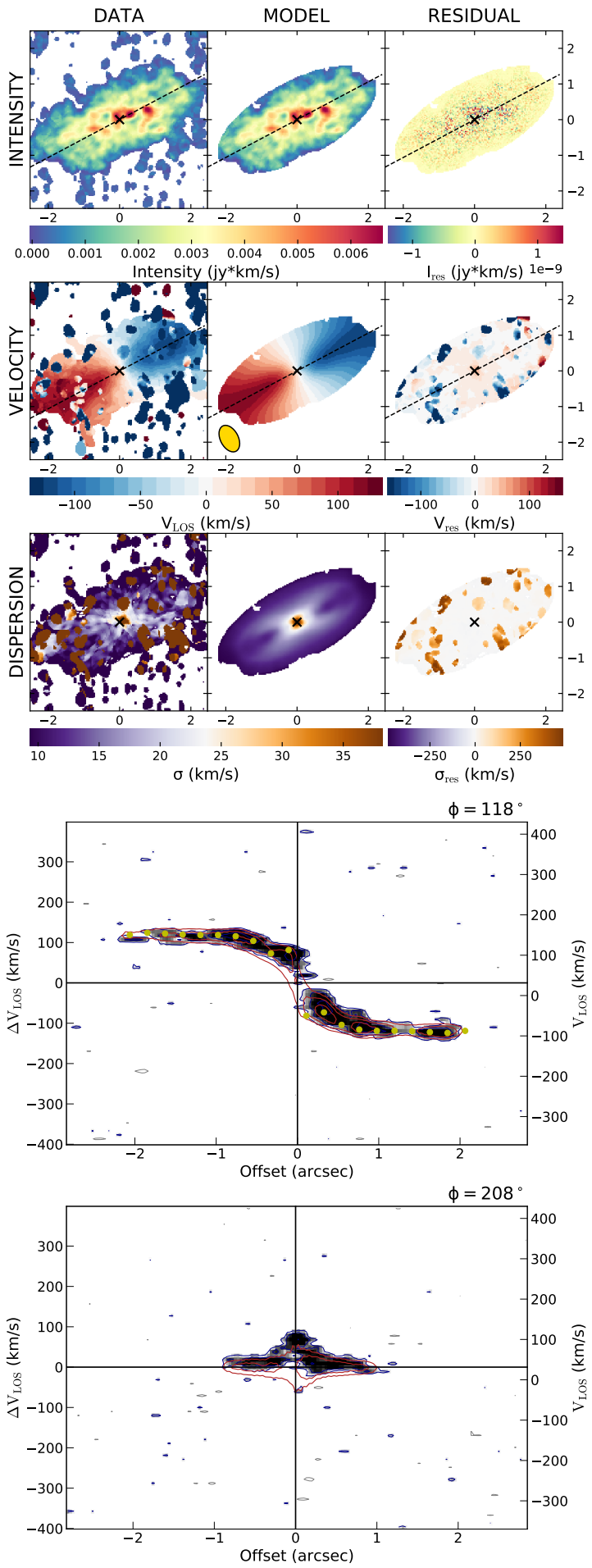

Fig. 3. Same as in Fig. 1 but for MCG-06-30-15. The synthesised beam size $\left(0.16^{\prime \prime} \times 0.13^{\prime \prime}\right)$ is plotted in yellow in the bottom left corner of the model velocity map with $\mathrm{PA}=118^{\circ}$. The contours level of the data and the model are at $[1,2,4,8,16,32,64]^{*} l$, where $l=0.00120941$.

axisymmetric model, DiskFit estimates the circular speed for kinematic data. For a non-axisymmetric model, DiskFit provides quantitative estimates of the non-circular flow speeds and an estimate of the mean circular speed when run on velocity fields.

Different axisymmetric and non-axisymmetric DiskFit kinematic models, such as pure rotation (flat disc), pure rotation (flat disc with warp), rotation plus radial motion, lopsided, and 
NGC 4968: pure rotation (flat disk)
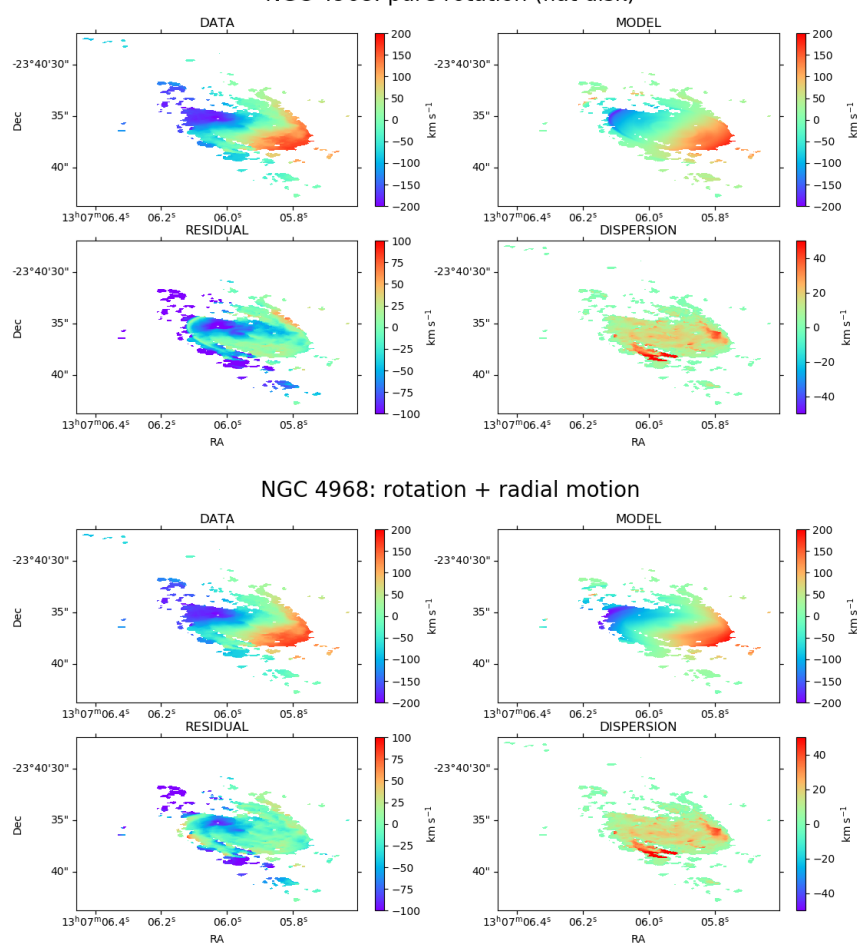

NGC 4968: non-axisymmetric model (bisymmetric, $m=2$ )
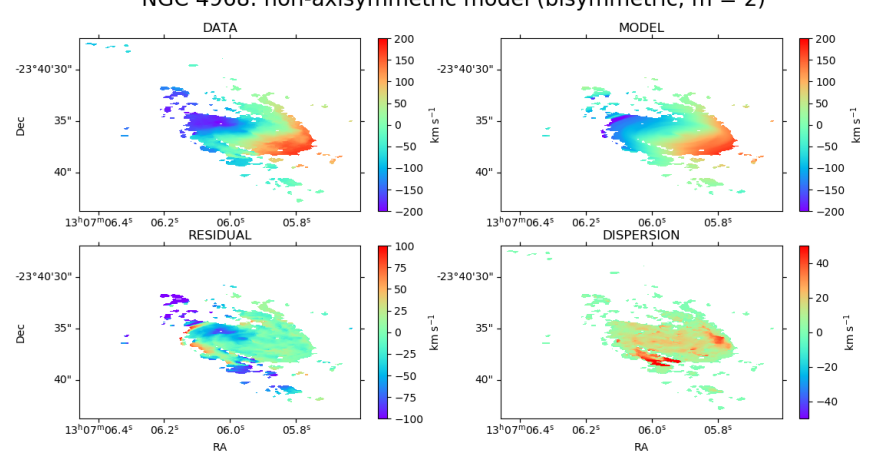

Fig. 4. Diskfit results for NGC 4968. Top panels: pure rotation DiskFit model(flat disc). Middle panels: rotation plus radial motion DiskFit model. Bottom panels: bisymmetric DiskFit model. For every panel, the top left figure reports the data, the top right represents the model, the bottom left the residual obtained subtracting the model from the data, and the bottom right figure the dispersion.

bisymmetric models are shown in Figs. 4-6 for NGC 4968, NGC 4845, and MCG-06-30-15, respectively.

\section{4. $C O(2-1)$ residual emission}

In the following sections, we present the properties and kinematics of the residual (both from ${ }^{3 \mathrm{D}} \mathrm{BAROLO}$ and DiskFit) $\mathrm{CO}$ molecular gas emission.

\subsection{Residuals from $3 D$ BAROLO}

As explained in Sect. 3, 3D Barolo makes a tilted-ring rotating disc model of the input data, hence if there are non-circular motions such as outflows, the model is not able to fully reproduce the observations. The non-circular motions could be misinterpreted as tilted rings of the plane. Some non-circular motions can be isolated from the rotating disc simply by subtracting
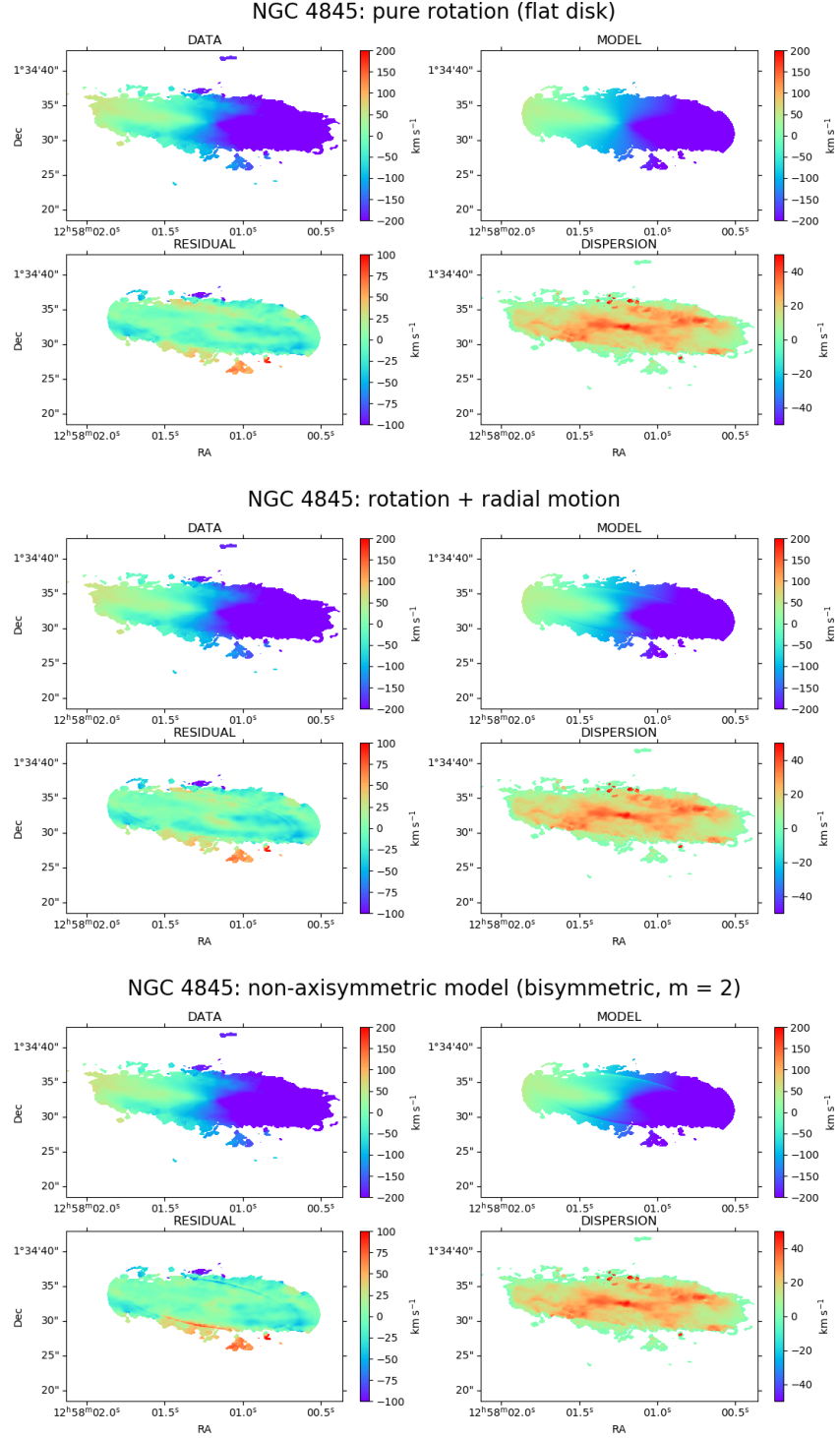

Fig. 5. DiskFit results for NGC 4845. Top panels: pure rotation DiskFit model (flat disk). Middle panels: rotation plus radial motion DiskFit model. Bottom panels: bisymmetric DiskFit model. For every panel, the top left figure reports the data the top right represents the model, the bottom left the residual obtained subtracting the model from the data, and bottom low right figure the dispersion.

the regular rotation pattern from the observed kinematics (observation-rotating disc model).

To see whether there are significant residuals in the $\mathrm{CO}(2-1)$ motions, we calculate the residuals in the kinematic maps (see Fig. 1 for NGC 4968, Fig. 2 for NGC 4845, and Fig. 3 for MCG06-30-15) by subtracting the $3 \mathrm{D}$ data cube produced by the model from the observations (the input continuum-subtracted $\mathrm{CO}(2-1)$ emission line data cube).

The 3D BAROLO model reproduces the observations of NGC 4968 relatively well along the kinematic major axis, leaving important residuals mainly in the north-east direction of the kinematic minor axis, as revealed by the velocity map in Fig. 1. The residuals reach a maximum velocity of $\sim 90 \mathrm{~km} \mathrm{~s}^{-1}$, that is blue-shifted to the south and south-east of the minor axis and red-shifted to the north and north-west of the minor axis with a velocity of $\sim 160 \mathrm{~km} \mathrm{~s}^{-1}$. Therefore, the maximum residual 

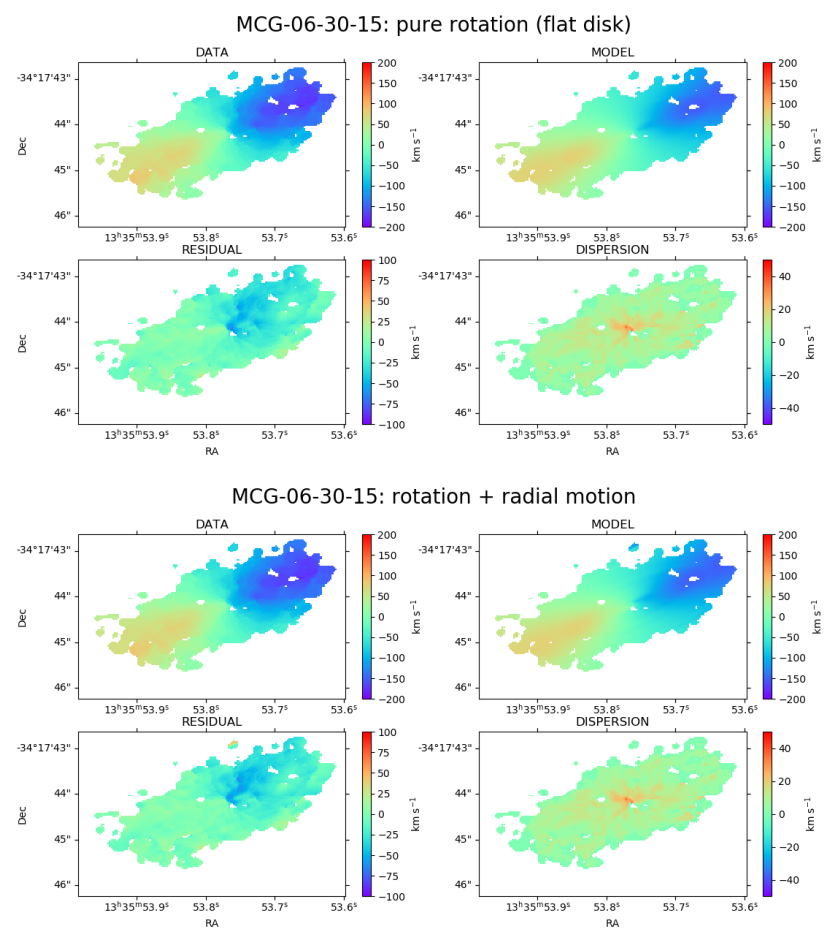

Fig. 6. DiskFit results for MCG-06-30-15. Top panels: pure rotation DiskFit model (flat disc). Bottom panels: rotation plus radial motion DiskFit model. For every panel, the top left figure reports the data, the top right represents the model, bottom left the residual obtained subtracting the model from the data, and the bottom right figure the dispersion.

velocity is the mean of the two components as we read them from the residual data cube $\sim 125 \mathrm{~km} \mathrm{~s}^{-1}$.

For NGC 4845, the model reproduces the observation reasonably well in the kinematic major axis, leaving almost no residuals. However, the model leaves small residuals in the nuclear region and either sides of the kinematic minor axis (see Fig. 2). The residuals reach a maximum velocity of $\sim 60 \mathrm{~km} \mathrm{~s}^{-1}$, that is blue-shifted to the north-east of the major axis, south and south-east of the kinematic minor axis and red-shifted to the north and north-west of the minor kinematic axis with a velocity of $\sim 50 \mathrm{~km} \mathrm{~s}^{-1}$. The maximum residual velocity turns out to be in this case $\sim 55 \mathrm{~km} \mathrm{~s}^{-1}$.

For MCG-06-30-15, the model reproduces well the observed $\mathrm{CO}(2-1)$ kinematics in the nuclear region and along both the kinematic minor and major axes (see Fig. 3). The residuals reach a maximum velocity of $\sim 20 \mathrm{~km} \mathrm{~s}^{-1}$, that is blue-shifted to the east direction with a velocity of $\sim 51 \mathrm{~km} \mathrm{~s}^{-1}$ and red-shifted to the south and south-west of the minor axis with a velocity of $\sim 20 \mathrm{~km} \mathrm{~s}^{-1}$. The maximum residual velocity is $\sim 35 \mathrm{~km} \mathrm{~s}^{-1}$.

Comparing these residual velocities of the gas in each galaxy with the velocities the regular rotation shows us the importance of the residual velocity for each galaxy, which could be a result of deviation from circular motions. For NGC 4968, the gas rotates at $\sim 200 \mathrm{~km} \mathrm{~s}^{-1}$ and there is a residual of $\sim 125 \mathrm{~km} \mathrm{~s}^{-1}$, which is a high fraction of the rotation velocity.

In NGC 4845, the rotational velocity of the gas is $\sim 200 \mathrm{~km} \mathrm{~s}^{-1}$ (taking the average of the two components using the input ALMA data cube), and a residual with a velocity of $\sim 55 \mathrm{~km} \mathrm{~s}^{-1}$ is detected. In MCG-06-30-15, the CO rotation velocity is $\sim 200 \mathrm{~km} \mathrm{~s}^{-1}$ and the residual velocity $\sim 35 \mathrm{~km} \mathrm{~s}^{-1}$, which is small with respect to the regular rotation velocity.
For all galaxies the residual velocities are smaller than the main rotation velocity, indicating that it is very unlikely that noncircular kinematics are tracing powerful outflows whether the cause is stellar or AGN related. Of all, the residual velocity of NGC 4968 is higher than the other two galaxies with respect the rotation velocity. The least residual velocity is found for MCG, indicating that the kinematics of the gas in the central $1 \mathrm{kpc}$ scale of this galaxy is best described by circular motions.

\subsection{Residuals from DiskFit}

As shown by the kinematic maps in Figs. 4-6 for NGC 4968, NGC 4845, and MCG-06-30-15, respectively, different DiskFit models reproduce the kinematics of the molecular gas as outlined by the $\mathrm{CO}(2-1)$ emission. Bisymmetric DiskFit models reproduce the observation much better than pure rotation DiskFit models for NGC 4968 and we consider this model as the best fit. The $\chi^{2}$ reduces by $\sim 50 \%$ for the radial motion model and by $\sim 63 \%$ for bisymmetric model with respect to its value for the pure rotation (flat disk) model, indicating that bisymmetric model is the best physical model for the kinematics of the gas in the central region of NGC 4968. This leads us to conclude that the residuals seen in the pure rotation models (see Fig. 4) cannot be ascribed to additional velocity components that do not belong to the rotational kinematics. However, there are still residuals left over from the best-fit model, mainly in the south direction of the minor kinematic axis and west of the major axis (see Fig. 4). Also the model rotation plus radial motion DiskFit reduces the residual observed in the pure rotation DiskFit model (see rotation plus radial motion DiskFit model in Fig. 4, middle panels), and indicates the presence of radial motions in the nuclear region of this galaxy.

Similarly, for NGC 4845, the pure rotation model reproduces the observation well (see top panels in Fig. 5); however, the bisymmetric model performs slightly better, leaving comparably smaller residuals in the kinematic minor axis (see bottom panels in Fig. 5). In this case the change in $\chi^{2}$ is small when bisymmetric flows are included, reducing $\chi^{2}$ by $\sim 30 \%$. We consider, however, that the bisymmetric model is the best-fit model.

Pure rotation (flat disk) DiskFit model reproduces the observation well for MCG-06-30-15, leaving only very small residuals (see top panels in Fig. 6), and this can be considered the best-fit model for this galaxy. For MCG-06-30-15, unlike NGC 4968, incorporating a radial component into a pure rotation DiskFit model (rotation plus radial motion model) does not reduce the residuals observed in the pure rotation model, indicating the absence of significant radial motions in the nuclear region of this galaxy.

\section{Line luminosity and molecular gas mass estimates}

To estimate the line luminosity $L_{\mathrm{CO}}^{\prime}$ and the molecular gas mass in the disc $M\left(\mathrm{H}_{2}\right)$, we present the profile of the integrated $\mathrm{CO}(2-1)$ emission line in Fig. 7. The line profiles are generally smooth enough to be well fitted with a single (NGC 4968) or two (NGC 4845 and MCG-06-30-15) Gaussian profiles. We integrate the line emission over the moment 0 maps. The results of the best-fit parameters are given in Table 3 together with the size of the region over which the line flux is estimated. This was chosen by selecting those pixels where the $\mathrm{S} / \mathrm{N}$ is larger than 3 , roughly corresponding to $10 \%$ of the maximum values. Then we derive the emission line luminosity $L_{\mathrm{CO}}^{\prime}$ using the relation given 

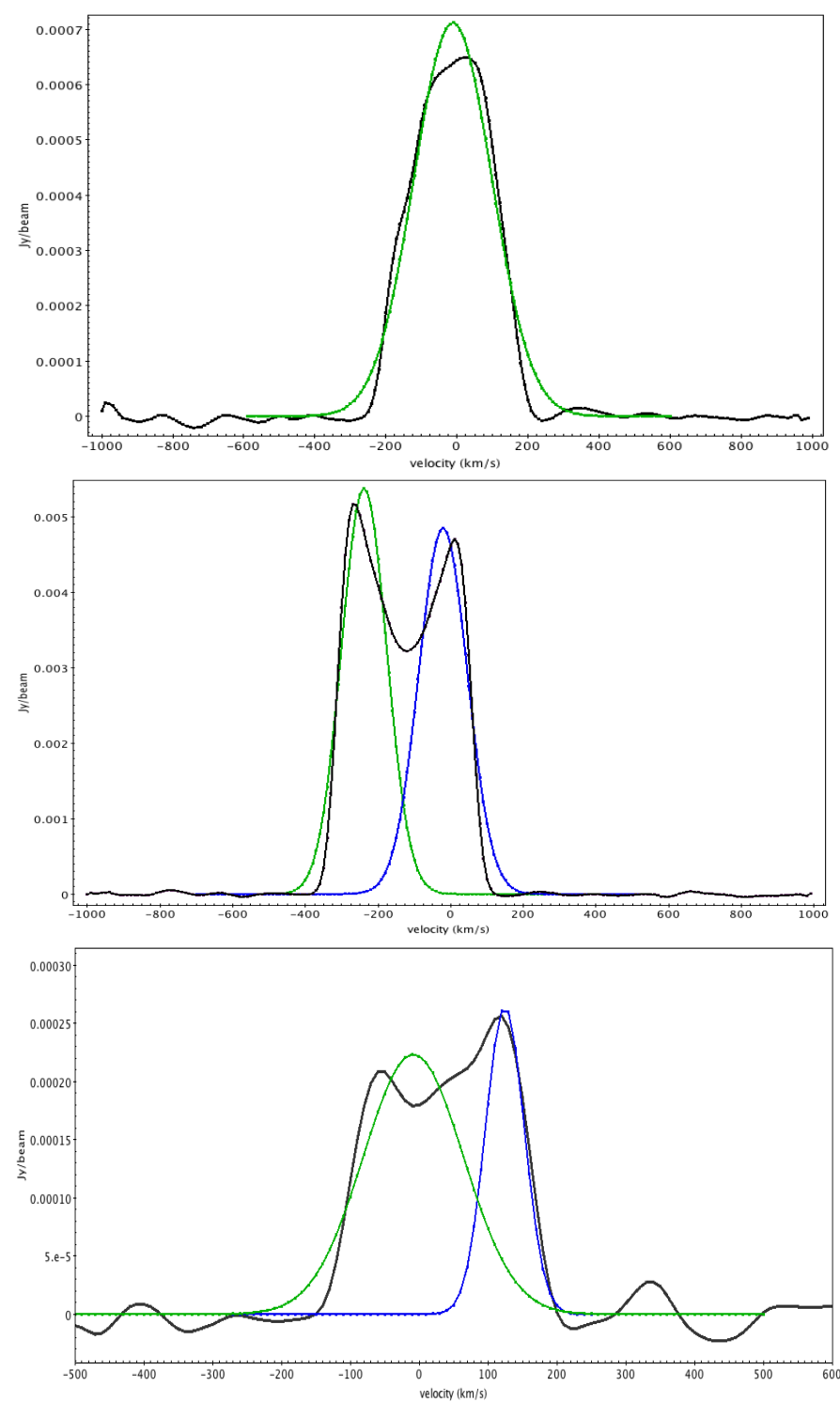

Fig. 7. Gaussian fit for the profile line of NGC 4968 (top panel), NGC 4845 (middle panel), and MCG-06-30-15 (bottom panel). Data are shown by the black line, while the fitted components by the blue and green lines (see detail in Table 3).

by Solomon \& Van de Bout (2005), that is

$L_{\mathrm{CO}}^{\prime}=3.25 \times 10^{7} S_{\mathrm{CO}} \Delta v v_{\mathrm{obs}}^{-2} D_{\mathrm{L}}^{2}(1+z)^{-3}$,

where $L_{\mathrm{CO}}^{\prime}$ is the $\mathrm{CO}$ line luminosity given in $\mathrm{K} \mathrm{km} \mathrm{s}^{-1} \mathrm{pc}^{2}$, $S_{\mathrm{CO}} \Delta v$ is the velocity-integrated flux in $\mathrm{Jy} \mathrm{km} \mathrm{s}^{-1}, v_{\mathrm{obs}}$ is the observed frequency in $\mathrm{GHz} ; z$ is the redshift, and $D_{\mathrm{L}}$ is the luminosity distance in $\mathrm{Mpc}\left(D_{\mathrm{L}}=D_{\mathrm{A}}(1+z)^{2}, D_{\mathrm{A}}\right.$ is the angular size distance) is the luminosity distance in Mpc. The molecular mass, $M\left(\mathrm{H}_{2}\right)$ is estimated using the relation (Solomon \& Van de Bout 2005)

$M\left(\mathrm{H}_{2}\right)=\alpha_{\mathrm{CO}} L_{\mathrm{CO}}^{\prime}$,

where $\alpha_{\mathrm{CO}}$ is the CO-to- $\mathrm{H}_{2}$ conversion factor. The value of $\alpha_{\mathrm{CO}}$ is highly uncertain; it depends on metallicity and environment (see i.e. Bolatto et al. 2013) and may vary in the range $0.8-3.2$. For simplicity we use the lower value, which is the average value in active galaxies. Furthermore, strictly speaking the conversion factor entails the $\mathrm{CO}(1-0)$ line luminosity;
Table 3. Results of a Gaussian fit to the total $\mathrm{CO}(2-1)$ spectrum.

\begin{tabular}{llll}
\hline \hline Parameters & NGC 4968 & NGC 4845 & MCG-6-30-15 \\
\hline$\mu_{\mathrm{v}}(1)$ & $-11 \pm 3$ & $-21 \pm 4$ & $125 \pm 3$ \\
$\mu_{\mathrm{v}}(2)$ & & $-238 \pm 4$ & $-8 \pm 8$ \\
$S_{\text {peak }}(1)$ & $0.71 \pm 0.01$ & $4.85 \pm 0.22$ & $0.26 \pm 0.03$ \\
$S_{\text {peak }}(2)$ & & $5.38 \pm 0.22$ & $0.22 \pm 0.01$ \\
$\sigma_{\mathrm{v}}(1)$ & $258 \pm 6$ & $157 \pm 10$ & $67 \pm 9$ \\
$\sigma_{\mathrm{v}}(2)$ & & $146 \pm 9$ & $171 \pm 21$ \\
\hline Area & $3^{\prime \prime} \times 1.4^{\prime \prime}$ & $13^{\prime \prime} \times 3^{\prime \prime}$ & $3.4^{\prime \prime} \times 1.2^{\prime \prime}$ \\
$S_{\mathrm{CO}(2-1)}$ & 36 & 617 & 16 \\
$L_{\mathrm{CO}(2-1)}^{\prime}$ & $4 \times 10^{7}$ & $12 \times 10^{7}$ & $1.3 \times 10^{7}$ \\
$M_{\text {tot }}\left(\mathrm{H}_{2}\right)$ & $3 \times 10^{7}$ & $9 \times 10^{7}$ & $1 \times 10^{7}$ \\
$M_{\text {res }}\left(\mathrm{H}_{2}\right)$ & $1 \times 10^{7}$ & $0.3 \times 10^{7}$ & $0.07 \times 10^{7}$ \\
\hline & & &
\end{tabular}

Notes. Top panel: best-fit parameters of the Gaussian model components shown in Fig. 7: central velocity $\left(\mu_{\mathrm{v}}\right)\left[\mathrm{km} \mathrm{s}^{-1}\right]$, flux amplitude $\left(S_{\text {peak }}\right)[\mathrm{mJy}]$, and velocity dispersion $\left(\sigma_{\mathrm{v}}\right)\left[\mathrm{km} \mathrm{s}^{-1}\right]$. The number in the parenthesis indicates the Gaussian components. Bottom panel: area over which the line emission is integrated on the collapsed image, the total $\mathrm{CO}(2-1)$ line flux $\left(S_{\mathrm{CO}}\right)\left[\mathrm{Jy} \mathrm{km} \mathrm{s}^{-1}\right]$, total $\mathrm{CO}(2-1)$ line luminosity $\left(L_{\mathrm{CO}}^{\prime}\right)\left[\mathrm{K} \mathrm{km} \mathrm{s}^{-1} \mathrm{pc}^{2}\right]$, and molecular gas mass $M\left(\mathrm{H}_{2}\right)\left[M_{\odot}\right]$. The molecular mass values corresponding to $\alpha=0.8$ are listed.

this means that in absence of similar $\mathrm{CO}(1-0)$ observations an assumption on the excitation status of the molecular gas must be made. We use the average $\mathrm{CO}$ spectral line distribution of Kamenetzky et al. (2016) corresponding to the $\log \left(L_{\mathrm{FIR}}\right)$ luminosity range $10-10.5 \log L_{\odot}$ to convert from the observed $L_{\mathrm{CO}(2-1)}^{\prime}$ to $L_{\mathrm{CO}(1-0)}^{\prime}$, which is used in Eq. (2). The resulting ratio is $\frac{L_{\mathrm{CO}(2-1)}^{\prime}}{L_{\mathrm{CO}(1-0)}^{\prime}}=2 / 3$. For NGC 4968 we use the ratio measured by Strong et al. (2004).

The total gas mass $M_{\mathrm{tot}}\left(\mathrm{H}_{2}\right)$ in the disc of the three galaxies is listed in Table 3, which agrees well with the typical values for other nearby and low-luminosity AGNs; these values are in line with the claim that Seyfert 2 galaxies seem to possess more molecular mass than Seyfert 1 galaxies (see Strong et al. 2004 and references therein) and agree with the single dish values published by Strong et al. (2004) for NGC 4968 and MCG-06-30-15 and by Rosario et al. (2018) for MCG-06-30-15.

Similarly, we determine the line luminosity and molecular gas mass in the modelled disc of each galaxy. The molecular gas mass fraction in the modelled disc of NGC 4968, NGC 4845, and MCG-06-30-15 corresponds to $\sim 69 \%, \sim 97 \%$, and $\sim 92 \%$ of the total molecular gas mass in each galaxy, respectively.

In addition to the total molecular gas mass and gas mass in the main rotating disc, we also estimated the line luminosity and then the molecular mass of the residuals for each galaxy using Eqs. (2) and (3) and listed in Table 3. The residuals are the emissions resulting from the substraction of the modelled from the observed data cube.

\section{Gas and continuum distribution}

Figure 8 shows the overlay of the collapsed CO intensity map with the map of the continuum at the observing frequencies, derived from the flux averaged over all the four spectral windows once neglecting the channels with the line emission. $\mathrm{CO}$ emission is absent from the centre of NGC 4968 and present in the other two galaxies. Together with the kinematical analysis discussed above we can argue that this result can be linked to the presence of the bar in the inner part (see Fig. 10). Indeed, 
the torque is positive inside (meaning the gas moves outwards) and negative outside (the gas flows inwards), which could be the reason there is no CO in the centre of NGC 4968.

The continuum distribution is overall more compact than the CO emission, especially in NGC 4968 and MCG-06-30-15. We may argue that some flux could be lost because of the high resolution of these observations and the lack of additional compact array observations. However because of the integrated $\mathrm{CO}$ flux agrees with that measured with single dish observations (Strong et al. 2004; Rosario et al. 2018), at least in NGC 4968 and MCG-06-30-15, the amount of missing flux should not be significant.

The origin of the majority of the continuum emission is very likely due to dust. The central nucleus is expected to emit synchrotron emission from the black hole jet or corona, which may have a significant contribution to the radiation in the millimetre range. However, the three galaxies are strong far-IR emitters as detected by infrared satellites (see i.e. Gruppioni et al. 2016; Cortese et al. 2014; Meléndez et al. 2014) and have a radio spectrum typical of a central synchrotron source with a spectral index decreasing with frequencies as $v \sim-0.6 \div-0.8$ (radio data taken from Condon et al. 2002; Mundell et al. 2009), which contributes a few percent at $1 \mathrm{~mm}$ wavelength.

\section{Discussion}

\subsection{Kinematical perturbations}

In addition to the kinematic maps, a comparison of the $p-v$ diagrams of the host galaxy disc with the fitted ${ }^{3 \mathrm{D}}$ BAROLO model of a rotating disc along the kinematic major and minor axes (see bottom panels in Figs. 1-3) further shows how well the model fits the data and helps to see the presence of any deviation from circular motions. For NGC 4968, along the kinematic major axis, the 3D BAROLO model with the rotating disc fits the rotation curve of the host galaxy disc relatively well, leaving little room for additional significant kinematical components (see the major axis in the bottom panel in Fig. 1). The 3D BAROLO rotating disc model also fits well the observation in the nuclear region and along the kinematic minor axis of the host galaxy disc; however, this leaves an important deviation at the end of the kinematic minor axis (see the minor axis in the bottom panel in Fig. 1), which agrees with what is observed in the corresponding velocity map in the same figure.

In NGC 4845, the 3D BAROLO model with the rotating disc fits the rotation curve of the host galaxy disc along the kinematic major axis (see the $p-v$ diagram in Fig. 2). Along the kinematic minor axis the 3D BAROLO model also fits the observations relatively well. However it fails around the kinematic minor axis; we see small deviations (see $p-v$ diagram in Fig. 2).

In MCG-06-30-15, the rotating disc model fits the rotation curve of the host galaxy disc very well along the kinematic major and minor axes, presenting only very small deviations (see the $p-v$ diagram in Fig. 3), further strengthening what is observed in the corresponding kinematic map (Fig. 3).

Deviations from circular motions could be caused by different mechanisms, such as outflows driven by AGN or star formation. Also, it has been known that non-circular motions of molecular gas in the nuclear regions of disc galaxies could be due to the existence of a bar-like structure, a warped circumnuclear disc, or radial motion resulting from other mechanisms. For example, using lower resolution $\mathrm{CO}(2-1)$ observations, Schinnerer et al. (2000) observed non-circular motions caused by a warped structure in the nuclear region (approximately
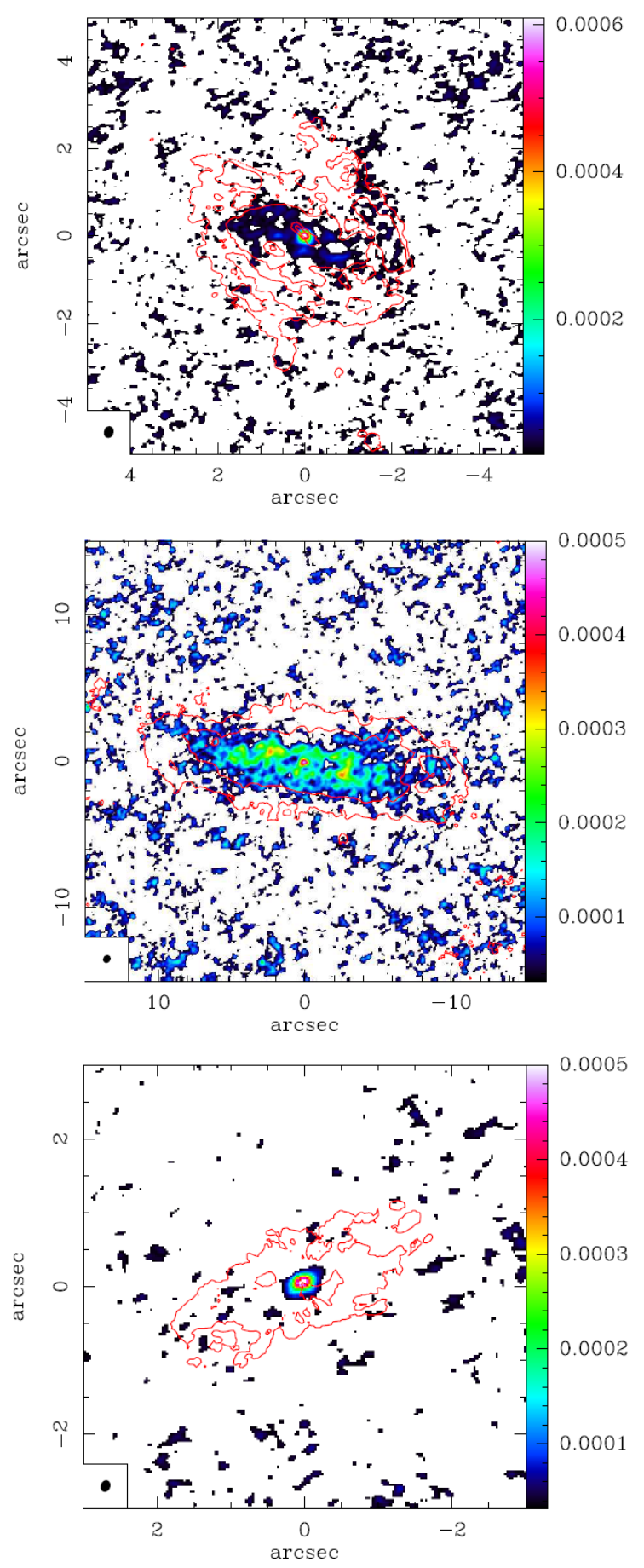

Fig. 8. Overlay of $\mathrm{CO}(2-1)$ contours on the $1.2 \mathrm{~mm}$ continuum image of NGC 4968 with 2 CO contours of 4 and $16 \%$ of maximum (top panel), NGC 4845 with 2 CO contours of 5 and $20 \%$ of maximum (middle panel), and MCG-06-30-15 with 2 CO contours of 5 and $20 \%$ of maximum (bottom panel). The continuum scale (colour wedge) is in $\mathrm{Jy} \mathrm{beam}^{-1}$. The $3 \sigma$ level is $0.1 \mathrm{mJy}$, therefore, $0.0001 \mathrm{Jy}^{\text {beam }}{ }^{-1}$ in the units of the figure for N4968; $0.5 \mathrm{mJy} \mathrm{beam}^{-1}$, then $0.0005 \mathrm{Jy}_{\text {beam }}{ }^{-1}$ for N4845; and $0.05 \mathrm{Jy} \mathrm{beam}^{-1}$, therefore, 0.00005 for MCG-06-30-15.

radial distances of $\left.0.7-1^{\prime \prime}\right)$ of NGC 3227 . Furthermore, it has been shown that gas inflows could be a result of gravity torques from non-axisymmetric potentials in the central regions of galaxies, such as streaming motions along a bar (see e.g. Ruffa et al. 2019). 
The presence of residuals/deviations could be either a consequence of some gas components not included within the main rotating disc considered by the model (possibly due to a difference in the geometry with the main rotating disc), or a deviation in the kinematics of the gas from circular motion, or both. In the galaxies of this study the observed residuals could be an indication of the presence of deviations in the kinematics of the gas from circular motions, as shown by the kinematic maps and corresponding $p-v$ diagrams.

The bisymmetric Diskfit model performs better in reproducing the observations than other DiskFit models in NGC 4845 and NGC 4968, indicating that the kinematical perturbations in these two galaxies are more likely due to a bar pattern, whereas the pure rotation model is the best-fit model for MCG-06-30-15. We note that the bisymmetric DiskFit model describes an elliptical or a bar-like flow; it is not surprising that bisymmetric model appears to be the best-fit model in NGC 4968 and NGC 4845, since the galaxies are shown to be barred (see more below).

As revealed by both models, we argue that although circular motion is the dominant aspect of the kinematics in the molecular disc of all galaxies, there is clear evidence for the presence of non-circular motions in the nuclear regions of NGC 4968 and NGC 4845, mainly in NGC 4968, where non-circular motions appeared to be significant (see Sects. 4.1 and 4.2). However, the small width of the residual velocity (compared to the circular velocity) indicates the absence of energetic feedback from the central AGN and star formation in the nuclear regions of the galaxies. Also, the SFR in all galaxies is very low (see Table 1).

Table 1 also lists the morphological types from either NED or Hyperleda: N4968 has a strong bar and the torque analysis (see below) also confirms that. We show in Fig. 9 a possible model for the rotation curve in the central kiloparsec of the galaxy, built from its various components, the bulge, disc of stars and gas, black hole and dark matter. The latter components contribute negligibly; however, the dark matter is required to explain a flat rotation curve in the outer parts. The bulge mass was taken from the decomposition of the red image, used in the potential calculation, and calibrated with the rotation curve. The black hole mass has been assumed to be $7 \times 10^{6} M_{\odot}$, which is about $0.2 \%$ of the mass of the bulge, according to scaling relations (e.g. Kormendy \& Ho 2013). The bulge dominates the region of interest, for the observed molecular component. Thus the mass decomposition allows the computation of the precessing frequency $\Omega-\kappa / 2$, and the determination of Lindblad resonances. If we place the corotation just outside the bar, as it is frequently observed in barred spirals (e.g. Buta \& Combes 1996), then the pattern speed of the bar is $\Omega_{\mathrm{b}}=52 \mathrm{~km} \mathrm{~s}^{-1} \mathrm{kpc}^{-1}$, putting corotation at $3.5 \mathrm{kpc}$ and inner Lindblad resonance (ILR) at $R=300 \mathrm{pc}$, corresponding to the $\mathrm{CO}$ ring.

N4845 morphology has a nice peanut shape bulge, and this is well known to be due to a bar (e.g. Combes et al. 1990). From our analysis there is evidence of additional weak kinematics, but we cannot argue that this is due to the presence of the bar. Our kinematics analysis seems to confirm that unlike the bar in NGC 4968, that in NGC 4845 is unable to change significantly the regular rotation pattern of the molecular gas. This additional weak kinematics (see Figs. 2 and 5) could be due to gas inflowing or outflowing the central region but with the present data we do not have firm evidence of it.

\subsection{Torque computation for NGC 4968}

To determine whether the gas is driven outwards or inwards, a calculation of the bar torques is necessary (e.g. Audibert et al.
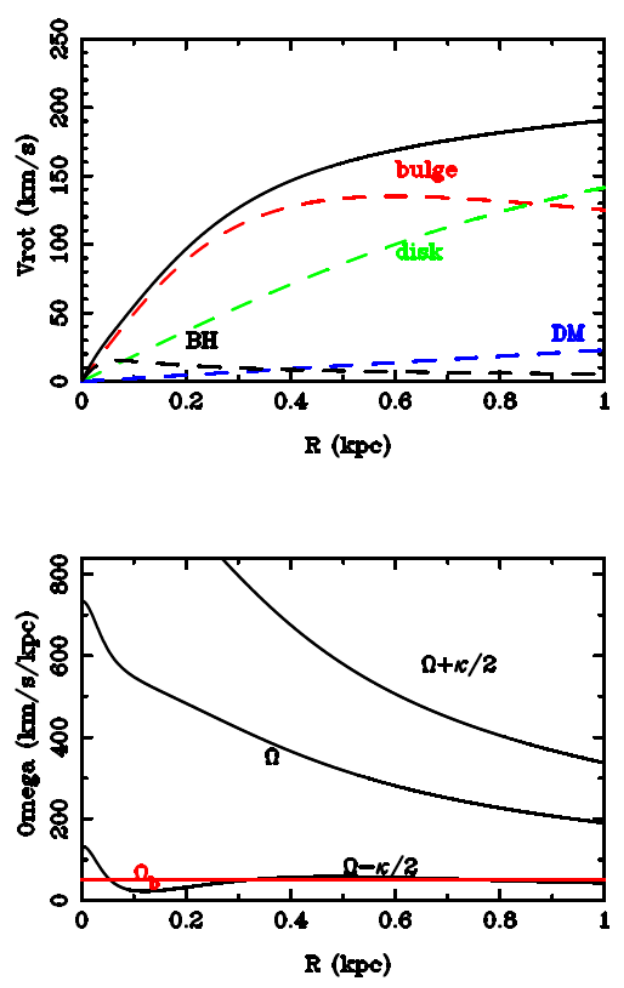

Fig. 9. Top panel: possible model for the rotation curve in the central kiloparsec of NGC 4948, built from its various components, that is, the bulge, disc of stars and gas, black hole, and dark matter. This latter contributes negligibly but it is required to explain a flat rotation curve in the outer parts (see text). Bottom panel: precessing frequency of elongated orbits, $\Omega-\kappa / 2$, used to model the orbits in the inner region of NGC 4968. The red line indicates the pattern speed of the bar (e.g. Buta \& Combes 1996).

2019). Through the Poisson equation, the gravitational potential is derived from the stellar density, which is assumed to be mainly responsible to the gravity forces in the plane of the galaxy inside the central kiloparsec. The dark matter is negligible in the very centre (e.g. NGC 4968 in Fig. 9). The stellar density is better traced by the HST $H$-band image $(F 160 W)$, since the dust impact is minimised in the near-infrared (NIR) (see Combes et al. 2019 for details). The HST-NIC2 image reveals a central bulge and a stellar disc. To de-project the galaxy to faceon, we first isolated the bulge, assumed to be spherical, which should not be de-projected. We then de-projected the galaxy disc with the adopted INC of $60^{\circ}$ and PA $250^{\circ}$. The bulge was then added to the de-projected image. We assumed a constant massto-luminosity ratio and calibrated it to retrieve the observed rotation curve, modelled as shown in Fig. 9. The gravitational potential is derived from the stellar distribution, assuming a thin disc of scale ratio $h_{z} / h_{\mathrm{r}}=/ 12$. The HST-NIR and $\mathrm{CO}(2-1)$ de-projected images of the galaxy were resampled to the same pixel size of 0.03 arcsec $=6 \mathrm{pc}$ (Combes et al. 2019).

We computed the bar gravity torques, following the method described in, for example García-Burillo et al. (2005) and Audibert et al. (2019). The forces are computed at each pixel by deriving the potential, and then the torques are computed on the gas, taking into account the gas density at the given pixel. The torque map is plotted in Fig. 10, together with the de-projected gas surface density, for comparison. As shown in the top panel of the figure, the torque map reveals the expected butterfly diagram (four-quadrant pattern) in relation to the bar orientation 


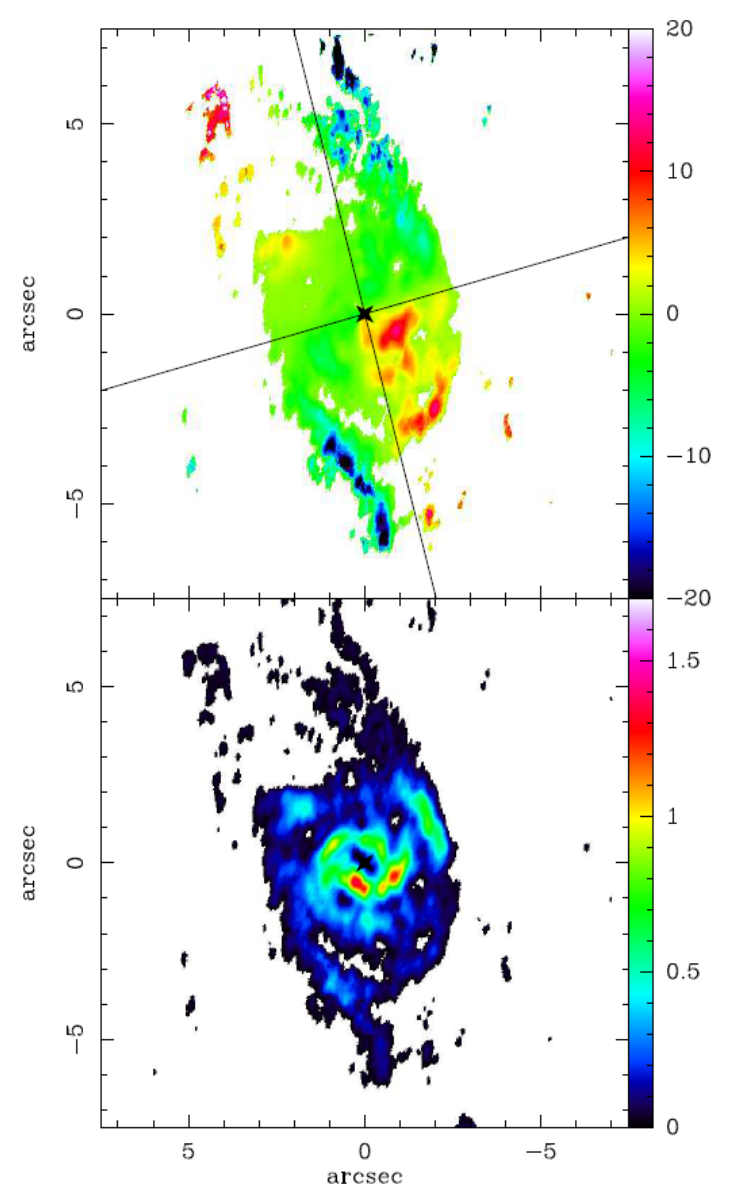

Fig. 10. Top panel: map of the gravitational torques exerted on the gas by the stellar potential in the centre of NGC 4968. The map shows in each pixel the torque derived from the HST-NIR image multiplied by the gas surface density. Both images have been de-projected to face-on (see text for details). The torques change sign as expected in a fourquadrant pattern (or butterfly diagram). The orientation of the quadrants follows the bar orientation. In this de-projected picture, the major axis of the galaxy is orientated parallel to the horizontal axis. Bottom panel: de-projected image of the $\mathrm{CO}(2-1)$ emission, at the same scale and with the same orientation, for comparison.

(indicated by straight lines) with torques changing sign in each quadrant. This implies that the observed non-circular motions are well fitted by the gas flow in a bar potential.

From the torque map we computed the azimuthal averages and obtain the effective torque at each radius per unit mass. This yields the derivative of the gas angular momentum at this radius. To derive the relative variation of angular momentum, we divided by the average angular momentum at this radius (from the rotation curve). This relative variation of angular momentum in one rotation is plotted in Fig. 11. The torque is positive in the very centre, meaning that the gas is driven outwards to the gas ring. Outside of the ring, the torque is negative and peaks at $\mathrm{d} L / L=-0.3$ in one rotation: the gas is driven inwards to accumulate in the ring. In this region the gas is losing about $30 \%$ of its angular momentum in one rotation.

The NLR dynamics does not uniquely show the presence of an outflow because it might be affected by extinction (Ferruit et al. 2000). If an ionised outflow were present, at least in the central region, the outward motions of the gas (as revealed by the positive torque in the very centre) could be partly interpreted as the interaction between the ionised outflows and the

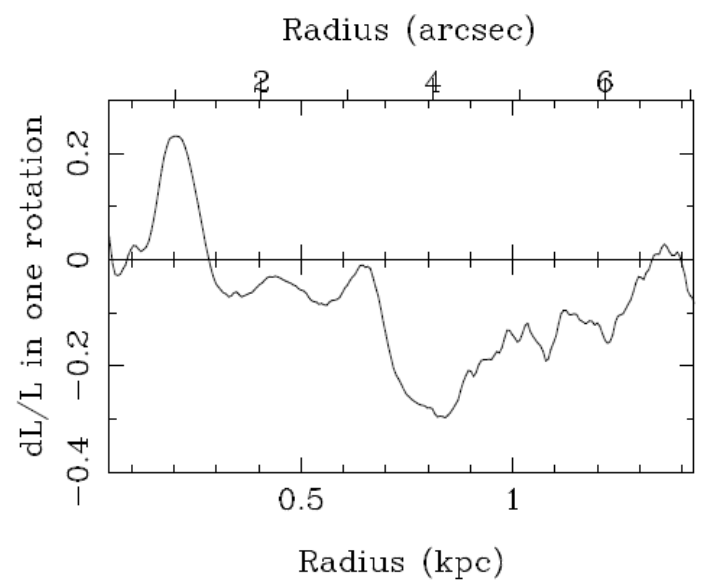

Fig. 11. Radial distribution of the average torque exerted by the stellar bar on the gas of NGC 4968. The torque is normalised to show the fraction of the angular momentum transferred from the gas in one rotation$\mathrm{d} L / L$, estimated from the $\mathrm{CO}(2-1)$ de-projected map. The curve is plotted only from a radius $=0.23 \operatorname{arcsec}$ (or $46 \mathrm{pc}$ ), which is the largest size of the beam.

gas. However, since the torque is negative in the outside of the ring, the outflows could be counter balanced by inward motions.

\section{Summary and conclusions}

We have analysed the properties and kinematics of the cold molecular gas in the nuclear and circumnuclear regions of three Seyfert galaxies, NGC 4968, NGC 4845, and MCG-06-30-15, using ALMA observations of their $\mathrm{CO}(2-1)$ emission line. We used the 3D BAROLO and DiskFit (both axisymmetric and nonaxisymmetric) software programs to model the kinematics of the molecular gas.

The main findings are summarised as follows:

- The intensity maps reveal a ring-like morphology in the nuclear regions of NGC 4968 and MCG-06-30-15, whereas the shape is edge-on in NGC 4845.

- The gas kinematics in the molecular discs of NGC 4845 and MCG-06-30-15 are dominated by pure rotational motion, but there is evidence for non circular-motions in NGC 4968 and weakly in NGC 4845.

- Unlike NGC 4845, where the deviation from circular motion is small, a significant non-circular motion is observed in NGC 4968, mainly along the kinematic minor axis, with a velocity of $\sim 115 \mathrm{~km} \mathrm{~s}^{-1}$, which is likely a consequence of the bar.

- Moreover, of all DiskFit models, the bisymmetric model is found to be the best-fit model for NGC 4968 and NGC 4845, in agreement with the nuclear bar origin.

- Regular rotation is shown to be the dominant kinematics of the gas in the nuclear region of MCG-06-3-15, and hence pure rotation model is the best-fit model for this galaxy.

- The molecular mass, $M\left(\mathrm{H}_{2}\right)$, in the nuclear disc is estimated to be $\sim 3-12 \times 10^{7} M_{\odot}($ NGC 4968$), \sim 9-36 \times 10^{7} M_{\odot}$ (NGC 4845), and $\sim 1-4 \times 10^{7} M_{\odot}$ (MCG-06-30-15), allowing the CO-to- $\mathrm{H}_{2}$ conversion factor $\alpha_{\mathrm{CO}}$ between 0.8 and 3.2, which is typical of nearby galaxies of the same type.

- The molecular gas mass of the modelled disc of each galaxy corresponds to $\sim 69 \%, \sim 97 \%$, and $\sim 92 \%$ of the total molecular gas mass in NGC 4968, NGC 4845, and MCG-06-30-15, respectively. 
- For the galaxy NGC 4968, placing the corotation just outside of the bar indicates a bar pattern speed of $\Omega_{\mathrm{b}}=52 \mathrm{~km} \mathrm{~s}^{-1} \mathrm{kpc}^{-1}$, putting corotation at $3.5 \mathrm{kpc}$ and the ILR ring at $R=300 \mathrm{pc}$, which corresponds to the $\mathrm{CO}$ ring. The computation of the torques exerted by the stellar bar on the gas shows that the torques are positive inside the molecular ring and negative outside, revealing that the gas is accumulating in the ILR. Thus, the observed non-circular motions in the molecular disc of NGC 4968 could be due to the presence of the bar in the nuclear region.

In summary, in the studied galaxies the radiative feedback or winds seen in the kinematics of emission lines from ionised gas (see Sect. 2) do not substantially alter the environments within molecular clouds that produce the bulk of the low-excitation $\mathrm{CO}$ emission. We do not find any strong evidence for gas kinematics close to the central AGN that might be univocally attributed to the effect of the AGN. The cold, star-forming molecular gas in the centre of the host galaxy is not strongly influenced by the presence of the AGN, even though these AGN are luminous enough to dynamically disturb this material. We cannot claim strong evidence in these sources of the long sought feedback/feeding effect owing to the coupling of mechanical energy from the nucleus with the cold star-forming phase.

Acknowledgements. This paper makes use of the following ALMA data: ADS/JAO.ALMA\#2017.1.00236.S.ALMA is a partnership of ESO (representing its member states), NSF (USA) and NINS (Japan), together with NRC (Canada), MOST and ASIAA (Taiwan), and KASI (Republic of Korea), in cooperation with the Republic of Chile. The Joint ALMA Observatory is operated by ESO, AUI/NRAO and NAOJ. The National Radio Astronomy Observatory is a facility of the National Science Foundation operated under cooperative agreement by Associated Universities, Inc. This publication has made use of data products from the NASA/IPAC Extragalactic Database (NED). We acknowledge the usage of the HyperLeda database (http://leda.univ-lyon 1.fr) A. Bewketu Belete acknowledges the support by the Brazilian National Council for Scientific and Technological Development (CNPq). R.S. acknowledges the support provided by CONICYT through FONDECYT postdoctoral research grant No. 3200909. JAFO and LS acknowledge financial support by the Agenzia Spaziale Italiana (ASI) under the research contract 2018-31-HH.0. Research activities of the Observational Astronomy Board of the Federal University of Rio Grande do Norte (UFRN) are supported by continuous grants from $\mathrm{CNPq}$, CAPES and FAPERN brazilian agencies. J.R.M. and A.B.B also acknowledge financial support from INCT INEspaço/CNPq/MCT. An anonymous referee is warmly thanked for their comments/suggestions have greatly improved the completeness of the paper.

\section{References}

Alonso-Herrero, A., Pereira-Santaella, M., García-Burillo, S., et al. 2018, ApJ, 859,144

Alonso-Herrero, A., García-Burillo, S., Pereira-Santaella, M., Davies, R. I., \& Combes, F. 2019, A\&A, 628, 65

Audibert, A., Combes, F., Garcia-Burillo, S., et al. 2019, A\&A, 632, A33

Bertola, F., Rubin, V. C., \& Zeilinger, W. W. 1989, ApJ, 345, L29

Bolatto, A., Wolfire, M., \& Leroy, A. K. 2013, ARA\&A, 51, 207

Brightman, M., \& Nanda, K. 2011, MNRAS, 413, 1206B

Buta, R., \& Combes, F. 1996, Fundam. Cosm. Phys., 17, 95

Carniani, S., Marconi, A., Maiolino, R., Balmaverde, B., \& Brusa, M. 2015 A\&A, 580, 102
Cicone, C., Maiolino, R., Sturm, E., Graciá-Carpio, J., \& Feruglio, C. 2014, A\&A, 562, A21

Combes, F., Debbasch, F., Friedli, D., Pfenniger, D., et al. 1990, A\&A, 233, 82 Combes, F., García-Burillo, S., Casasola, V., et al. 2013, A\&A, 558, A124

Combes, F., García-Burillo, S., Casasola, V., et al. 2014, A\&A, 565, A97

Combes, F., García-Burillo, S., Audibert, A., et al. 2019, A\&A, 623, A79

Condon, J. J., Cotton, W. D., \& Broderick, J. J. 2002, AJ, 124, 675

Cortese, L., Fritz, J., Bianchi, S., et al. 2014, MNRAS, 440, 942

Dasyra, K. M., \& Combes, F. 2011, A\&A, 533, L10

Dasyra, K. M., \& Combes, F. 2012, A\&A, 541, L7

Di Teodoro, E. M., \& Fraternali, F. 2015, MNRAS, 451, 3021

Domínguez-Fernández, A. J., Alonso-Herrero, A., García-Burillo, S., et al. 2020, A\&A, 643, A127

Fernández-Ontiveros, J. A., Dasyra, K. M., Hatziminaoglou, E., et al. 2020, A\&A, 633, A127

Ferruit, P., Wilson, A. S., Mulchaey, J., et al. 2000, ApJS, 128, 139

Feruglio, C., Maiolino, R., Piconcelli, E., et al. 2010, A\&A, 518, L155

Fiore, F., Feruglio, C., Shankar, F., Bischetti, M., \& Bongiorno, A. 2017, A\&A, 601, A143

Fluetsch, A., Maiolino, R., Carniani, S., et al. 2019, MNRAS, 483, 4586

García-Burillo, S., Combes, F., Schinnerer, E., et al. 2005, A\&A, 441, 1011

García-Burillo, S., Combes, F., Usero, A., et al. 2014, A\&A, 567, A125

García-Burillo, S., Combes, F., Usero, A., et al. 2015, A\&A, 580, A35

García-Burillo, S., Combes, F., Ramos Almeida, C., et al. 2016, A\&A, 823, L12

García-Burillo, S., Combes, F., Ramos Almeida, C., et al. 2019, A\&A, 632, A61 Gruppioni, C., Berta, S., Spinoglio, L., et al. 2016, MNRAS, 458, 4320 Irwin, J. A., Henriksen, R. N., Krause, M., et al. 2015, ApJ, 809, 172

Kamenetzky, J., Rangwala, N., Glenn, J., Maloney, P. R., \& Conley, A. 2016, ApJ, 829, 93

Kanekar, N., \& Chengalur, J. N. 2008, MNRAS, 384, L6

Kormendy, J., \& Ho, L. C. 2013, ARA\&A, 51, 511

LaMassa, S. M., Yaqoob, T., Levenson, N. A., et al. 2017, ApJ, 835, 91

Lutz, D., Sturm, E., Janssen, A., et al. 2020, A\&A, 633, A134

Makarov, D., Prugniel, P., Terekhova, N., Courtois, H., \& Vauglin, I. 2014, A\&A, 570, A13

Malkan, M. A., Gorjian, V., \& Tam, R. 1998, ApJS, 117, 25

McMullin, J. P., Waters, B., Schiebel, D., et al. 2007, ASP Conf. Ser., 376, 127

Meléndez, M., Mushotzky, R. F., Shimizu, T. T., Barger, A. J., \& Cowie, L. L. 2014, ApJ, 794, 152

Morganti, R. 2017, Front. Astron. Space Sci., 4, 42

Morganti, R., Oosterloo, T., Oonk, J. B. R., et al. 2015, A\&A, 580, A1

Mundell, C. G., Ferruit, P., Nagar, N., \& Wilson, A. S. 2009, ApJ, 703, 802

Oosterloo, T., Oonk, J. B. R., Morganti, R., Combes, F., \& Dasyra, K. 2017, A\&A, 608, A38

Peters, W., \& Kuzio de Naray, R. 2017, MNRAS, 469, 3541

Ramakrishnan, V., Nagar, N. M., Finlez, C., et al. 2019, MNRAS, 487, 444

Raimundo, S. I., Davies, R. I., Canning, R. E., et al. 2017, MNRAS, 464, 4227

Rosario, D. J., Burtscher, L., Davies, R. I., et al. 2018, MNRAS, 473, 5658

Ruffa, I., Davis, T. A., Prandoni, I., et al. 2019, MNRAS, 489, 3739

Rupke, D. S. N., \& Veilleux, S. 2013, ApJ, 768, 75

Rush, B., Malkan, M. A., \& Spinoglio, L. 1993, ApJS, 89, 1

Sakamoto, K., Aalto, S., Combes, F., Evans, A., \& Peck, A. 2014, ApJ, 797, 90

Salak, D., Nakai, N., Sorai, K., \& Miyamoto, Y. 2020, ApJ, 901, 151

Schinnerer, E., Eckart, A., \& Tacconi, L. J. 2000, ApJ, 533, 826

Schmitt, H. R., Donley, J. L., Antonucci, R. R. J., et al. 2003, ApJS, 148, 327

Sirressi, M., Cicone, C., Severgnini, P., et al. 2019, MNRAS, 489, 1927

Slater, R., Nagar, N. M., Schnorr-Müller, A., et al. 2019, A\&A, 621, A83

Solomon, P. M., \& Van de Bout, P. A. 2005, ARA\&A, 43, 677

Strong, M., Pedlar, A., Aalto, S., et al. 2004, MNRAS, 353, 1151

Tadhunter, C., Morganti, R., Rose, M., Oonk, J. B. R., \& Oosterloo, T. 2014 ,

Nature, 511, 440

Thomas, A. D., Dopita, M. A., Shastri, P., et al. 2017, ApJS, 232, 11

Wu, Y., Charmandaris, V., Huang, J., et al. 2009, ApJ, 701, 658

Veilleux, S., Maiolino, R., Bolatto, A., et al. 2020, A\&ARv, 28, 1 